Marquette University

e-Publications@Marquette

7-1-2014

Field Experiment Tests for Discrimination against Hispanics in the U.S. Rental Housing Market

Andrew Hanson

Marquette University, andrew.r.hanson@marquette.edu

Michael Santas

Georgia State University

Published version. Southern Economic Journal, Vol. 81, No. 1 (July 2014): 135-167 appears with permission of Southern Economic Association. DOI. (C) 2014 Southern Economic Association. Used with permission. 


\title{
Field Experiment Tests for Discrimination against Hispanics in the U.S. Rental Housing Market
}

\author{
Andrew Hanson* and Michael Santas†
}

This article tests for discrimination against Hispanics in the U.S. rental housing market using e-mail correspondence with landlords advertising units online. We divide Hispanics into two groups: those that appear assimilated into American culture and recent immigrants. We find little difference in the treatment of assimilated Hispanics and whites; however, Hispanics we portray as recent immigrants receive less favorable treatment with margins of net discrimination as large as $6.89 \%$ of landlords. We also find discrimination varies significantly at the region level and by the ethnic composition of neighborhoods.

\section{JEL Classification: J15, C93}

\section{Introduction}

The Hispanic share of the population of the United States grew from $12.5 \%$ in the 2000 Census to $16.7 \%$ in 2011 according to the U.S. Census Bureau. The growth of the Hispanic population represents more than half of all U.S. population growth in the decade between 2000 and 2010 and positions Hispanics as the largest minority group by a wide margin (African Americans are second with a $12.6 \%$ share of the population). Although growing as a proportion of the population, Hispanics tend to live in ethnically isolated areas, ${ }^{1}$ in housing units with worse quality, and in neighborhoods with worse public services and more crime than

* Department of Economics, Marquette University, P.O. Box 1881, Milwaukee, WI 53201, USA; E-mail andrew.r.hanson@marquette.edu; corresponding author.

$\dagger$ Department of Economics, Georgia State University, P.O. Box 3992, Atlanta, GA 30302, USA; E-mail msantas@gmail.com.

We would like to thank Cary Deck, Zackary Hawley, Juan Jose Miranda Montero, Geoffrey Turnbull, Kurt Schnier, Spencer Banzhaf, and seminar participants at the University of Wisconsin-Milwaukee, Universitat de Barcelona, Institut d'Economia, and American Real Estate and Urban Economics Annual Meeting for helpful discussion and comments. We would also like to thank Hal Martin and Jason Beall for outstanding research assistance. Two anonymous reviewers provided suggestions that improved the clarity and rigor of the document.

Received October 2012; accepted August 2013.

${ }^{1}$ The Dissimilarity Index, which measures the percentage of a group's population that would have to change residence for each neighborhood in a metropolitan area to have the same percentage of that group, ranges from 0.273 in St. Louis, MO, to 0.676 in Providence, RI, for Hispanics living in U.S cities (U.S. Census Bureau). This means that between $27.3 \%$ and $67.6 \%$ of Hispanic residents would have to change neighborhoods to make an even distribution of Hispanics across neighborhoods in U.S. cities. 
the white majority. ${ }^{2}$ Although there are many possible explanations for the inequality and isolation Hispanics face in the housing market, discrimination is one possible channel through which geographic segregation and economic inequality persists.

This article tests for discrimination against Hispanics in the rental housing market using a field experiment conducted across several U.S. cities. We consider discrimination in this context to be unequal treatment of potential tenants by landlords based on their perceived ethnicity. Studying housing discrimination against Hispanics in the rental market is especially important, given that the proportion of the Hispanic population that owns a home is $20 \%$ to $40 \%$ lower than whites according to the Department of Housing and Urban Development (HUD). Hispanics are also an understudied minority group relative to African Americans, with the latest audit-style experiments occurring more than 10 years ago: To our knowledge no study has used online search and e-mail correspondence to study discrimination against Hispanics. In addition, because Hispanics are not identifiable by appearance alone like some other minority groups, using e-mail communication is advantageous to signal ethnicity. E-mail also allows for adding a dimension of cultural assimilation that has not been used in previous studies of discrimination.

We test for discrimination in the rental housing market against Hispanics using an e-mail audit technique applied to advertisements from landlords using the online venue Craigslist (craigslist.org). Landlords posting units from 21 large MSAs (metropolitan areas) are randomly chosen to receive e-mail inquiries about their advertisement. The structure of our experiment is similar to Hanson and Hawley (2011) in that we use an audit-style field experiment, but we augment the design to account for two different groups of Hispanics. The first group signals that the potential tenant is a recent immigrant, while the second group is assimilated to American culture. Additionally, we vary the correspondence with landlords to offer other signals of tenant quality and cultural assimilation.

Previous studies such as Page (1995), Ondrich, Stricker, and Yinger (1998), Zhao (2005), and Zhao, Ondrich, and Yinger (2006) have examined discrimination against Hispanics using data based on in-person audit experiments. To our knowledge, this is the first study using an online field experiment to examine housing market discrimination against Hispanics. Heckman and Siegelman (1993) and Heckman (1998) critique studies based on in-person audits for having bias because they may be affected by unobserved characteristics of the actors. ${ }^{3}$ In addition, in-person auditors may prompt discriminatory behavior from agents or misreport treatment. Using automated e-mail communication, we avoid any potential problems with using actors to study discrimination. In addition, cultural assimilation can be portrayed through names and message quality. This is crucial to accurately capturing and more fully understanding any discrimination Hispanics encounter in their search for housing.

\footnotetext{
${ }^{2}$ Boehm and Schlottmann (2008), using data from the American Housing Survey, find Hispanics are more likely than whites to report major structural problems and interior deterioration in their housing units. They are also more likely to report having unsafe drinking water and poor quality heating. When asked about neighborhoods, Hispanics are more likely to report that crime is a problem, that police protection is inadequate, and that there are abandoned buildings close by.

${ }^{3}$ The Heckman critique influenced the design of the 2000 Housing Discrimination Study (HDS) audits to ensure collection of auditor traits. See Ross and Turner (2005) and Turner et al. (2002) for details on the 2000 HDS and how they limit bias from using actors.
} 
The results of our experiment paint two different pictures of discrimination against Hispanics. We find almost no evidence of discrimination against Hispanics that we portray as assimilated into American culture; this is true regardless of e-mail quality, ethnicity of neighborhood surrounding housing units, or unit characteristics. In fact, for some of these audits there is weak evidence landlords favor Hispanics over whites. When we portray Hispanics as being recent immigrants, we find net discrimination by $2.9 \%$ of landlords. When e-mail quality is low for this group, discrimination doubles to $5.8 \%$ of landlords, and when we consider positive response as the outcome of interest, rather than simply response/nonresponse, $6.89 \%$ of landlords practice unequal treatment. We also find differences in discrimination for this group by ethnic composition of neighborhood and type of unit, and that landlords practice more subtle forms of discrimination against this group through the language used in correspondence.

The remainder of the article begins by reviewing the previous literature on housing discrimination, with a focus on discrimination against Hispanics and using e-mail communication as a method to test for discrimination. Section 3 describes our experimental design. Section 4 highlights the characteristics of our sample, while section 5 discusses the main findings. The final section of the article concludes.

\section{Previous Studies of Housing Market Discrimination}

The primary method used to test for discrimination in the housing market is an audit, or matched pair field experiment. In an in-person audit study, two subjects (one from the majority racial group and one from the minority) are matched based on observable characteristics (excluding race) and trained how to act toward a real estate agent or landlord. The subjects are sent (in random order) to a landlord or real estate agent's office to inquire about an advertised housing unit. Typically subjects will report if they are shown the advertised unit, if they are shown similar units, how many additional units they are shown, and potentially several other objective measures of treatment. Audit field experiments have become a popular method for testing racial discrimination in the housing market. In theory, the only dimension the actors differ on is race, so that any difference in treatment is attributed to racial discrimination.

Studies of discrimination using data based on in-person audits include Yinger (1986), Page (1995), Ondrich, Stricker, and Yinger (1998), Ondrich, Ross, and Yinger (2003), Choi, Ondrich, and Yinger (2005), Ross and Turner (2005), Zhao (2005), and Zhao, Ondrich, and Yinger (2006). Yinger (1986) examines the Boston housing market using unique data; the other studies use data from the Housing Discrimination Studies (HDS) conducted by HUD. Page (1995) tests for differences in the number of homes real estate brokers show whites and minorities, finding that African Americans and Hispanics are shown 10\% to 20\% fewer homes. Ondrich, Stricker, and Yinger (1998) use in-person audits from several cities in the United States and estimate the probability of favorable treatment toward a white customer in a real estate office. Measures of favorable treatment include likelihood of a call back, questions about income, follow-up calls made, questions about household needs, financial assistance offered, whether or not they showed the auditor the advertised unit, and whether or not they told the auditor the unit was available. Using a fixed effects logit technique they calculate the probability of 
favorable treatment for whites, African Americans, and Hispanics. They find that whites on average have positive and statistically significant differences in the probability of being treated favorably compared to African Americans and Hispanics.

Ondrich, Ross, and Yinger (2003) focus on hypotheses of why discrimination in the housing market exists. Evidence of perceived customer preference discrimination is more likely to occur when the advertised unit is in a white neighborhood. Since many brokers may perceive that minorities prefer to live in neighborhoods with other minorities they are less likely to spend their time showing a unit that is in a white neighborhood. They do not find that one hypothesis dominates, but find that reasons for discrimination vary by neighborhood and cities. In general, they find some evidence that discrimination exists as a result of broker prejudice and white client prejudice.

Ross and Turner (2005) use data from a newer HDS and find results consistent with previous studies where discrimination exists against African Americans and Hispanics in rental and owner-occupied housing markets. However, Ross and Turner's study finds that in many cases the level of discrimination has declined substantially from previous levels in 1981. Overall, levels of discrimination have decreased by $8 \%$ to $12 \%$; however, specific types of discrimination have not improved and in some cases have risen. They point out that discrimination against Hispanics remains at about the same level. Furthermore, discrimination against Hispanics in terms of financial assistance has risen in every aspect considered in the study. Choi, Ondrich, and Yinger (2005) also use data from the $2000 \mathrm{HDS}$ and find that discrimination has declined since the 1989 HDS. Choi, Ondrich, and Yinger (2005) examine the causes of discrimination and conclude that it is primarily driven by agents' own preferences and through catering to white clients.

Zhao (2005) uses data from the 2000 HDS and finds that discrimination against blacks in the number of housing units shown increased between 1989 and 2000, while discrimination against Hispanics remained constant. Zhao's work suggests that the cause of discrimination is white customers' prejudice. Zhao, Ondrich, and Yinger (2006) focus on discrete choices by real estate brokers, such as whether to tell a customer that an advertised house is available. They find that discrimination remains a factor in housing markets but has declined between 1989 and 2000 , and that real estate broker prejudice and white customer prejudice are the primary causes of discrimination.

In-person audits have the advantage of directly revealing race or ethnicity to agents through skin color differences in a test for unequal treatment. They also have the advantage of an experimental design, as opposed to research that uses hedonic methods to uncover price differentials, that greatly reduces bias. In addition, in-person audits provide a venue that mimics the portion of the housing market where face-to-face interaction happens. While faceto-face interaction may be quite common in the home purchase market, the introduction of online listings and e-mail make electronic interaction a likely first step in many housing market transactions - especially in the rental market.

More recent work applies the audit technique to e-mail correspondence. As with the work presented here, these online audits use names to signal race or ethnicity and e-mail content to signal quality. The online audit technique is also advantageous because it eliminates possible actor bias that may occur when a study is not "double-blind" as actors inquiring about a unit for rent know they are part of a study about discrimination. Although actors in an audit study are trained to act in a specific manner, they may still exhibit traits or mannerism knowingly or unknowingly because of a preconceived belief about discrimination. Notably, the 2000 HDS 
actors were trained to behave similarly and instructed to report similar characteristics to agents, and reporting of treatment was also made standard.

Examples of e-mail audit studies are Ahmed and Hammarstedt (2008, 2009), Ahmed, Andersson, and Hammarstedt (2010), Bosch, Carnero, and Farre (2010), Hanson and Hawley (2011), and Baldini and Federici (2011). Ahmed and Hammarstedt (2008) focus on the housing market in Sweden and test for discrimination against Muslim minorities. Ahmed and Hammarstedt test for discrimination based on sexual orientation but do not consider race or ethnicity. Ahmed, Andersson, and Hammarstedt (2010) and Bosch, Carnero, and Farre (2010) study how the interaction between positive information and race affects landlord discrimination. Ahmed, Andersson, and Hammarstedt (2010) find that while information (including marital status, employment information, age, and education level) does increase the response rate for minority applicants, it does not decrease the difference in response rates between native Swedes and the Muslim minority. Bosch, Carnero, and Farre (2010) find discrimination against the Moroccan minority in Spain and that positive information increases the chance of being contacted but does not eliminate discrimination. Hanson and Hawley (2011) sample Craiglist in the United States and test for discrimination against African Americans in the rental housing market. Finally, Baldini and Federici (2011) uncover discrimination against Arabs/Muslims and Eastern Europeans in the Italian rental market.

There are two other studies we are aware of that use online housing market interactions in the United States to study discrimination-Carpusor and Loges (2006) and Ewens, Tomlin, and Wang (2012) - although neither uses an audit-style design so they cannot completely control for landlord characteristics or determine how often landlords treat auditors equally. Both studies examine discrimination against African Americans, and both find large positive levels of discrimination.

In addition to the more popular in-person and e-mail audits, there is a small literature that uses phone interview audits to examine discrimination. Phone interview audits, as in Massey and Lundy (2001) and Fischer and Massey (2004), rely on the dialect of individuals to signal race. Phone interview audits show substantial discrimination against African Americans who speak with "Black English Vernacular" and discrimination to a lesser extent against African Americans who speak in a linguistic style described as "Black Accented English"; the reference group in either case is treatment of whites speaking "Middle Class English."

Most of the previous literature is unable to definitively determine the source of racial discrimination in housing markets, and there has been a recent push to determine if discrimination happens because of agent/landlord personal preferences (referred to as animus or taste-based discrimination) or as a result of profit maximization with imperfect information (referred to as statistical discrimination). Ewens, Tomlin, and Wang (2012) give a lucid description of the two sources of discrimination, as well a formal model that helps to clarify how the sources can be distinguished. Although they conclude that distinguishing between the types of discrimination is difficult, they propose that varying an information signal with a race signal can work to separate the cause of discrimination if there are differences in the variance of signal quality between races along an observable characteristic (they use the percentage of African American residents in a neighborhood). ${ }^{4}$

\footnotetext{
${ }^{4}$ The Ewens, Tomlin, and Wang (2012) model and experiment are not directly applicable here, as they rely on use of fairly strong information signals about tenant desirability, like smoking preference, employment, and credit rating, while we use much weaker signals like language that may not be interpreted in the same manner by landlords.
} 


\section{Experiment Design}

We design our discrimination experiment as an audit, or within subjects' field experiment, conducted with e-mail. This means that each prospective landlord in our sample receives two e-mail inquiries - one from a tenant with a white name, and the other from a tenant with a Hispanic name. Our design follows the e-mail audit-style designs used by Ahmed and Hammarstedt (2008), Ahmed, Andersson, and Hammarstedt (2010), Bosch, Carnero, and Farre (2010), and Hanson and Hawley (2011).

The design of our field experiment is unique from previous studies of discrimination using Hispanics because we develop a technique specific to measuring unequal treatment based on the level of cultural assimilation. Discrimination against Hispanics is likely to occur for different reasons than for African Americans, as there are not necessarily differences between them and whites in physical appearance, but there may be differences in language, culture, and nationality. Since Hispanics are generally more recent immigrants than other ethnic groups they do not have a long history of shared nationality with white Americans and may be more likely to be perceived as outsiders. In addition, Hispanics make up $80 \%$ of the estimated undocumented workers in the United States (Ditlmann et al. 2010), something that is currently a source of tension in national politics. In a 2007 survey, $62 \%$ of Americans indicated that the issue of illegal immigration is very important to them (Ditlmann et al. 2010), highlighting a potential source of animosity between whites and Hispanics. If landlords perceive an inquiry may be from an illegal immigrant, they may be more likely to discriminate, either because of their own preferences or because of preferences they perceive about their other clients.

Hispanics that have lived in the United States for the majority of their lives or were born in the United States are generally more assimilated to American culture. Assimilated Hispanics may face racial or ethnic discrimination but are less likely to be discriminated against because of language and cultural barriers. Furthermore, assimilated Hispanics may be less likely to be perceived as illegal immigrants. These sources of discrimination against Hispanics suggest a two-group structure for the experimental design, which we achieve through variation in constructing the names associated with potential tenants and the text of the inquiry we use to contact landlords.

As with previous discrimination studies conducted via e-mail, we reveal potential tenant ethnicity through the name associated with the inquiry. We reveal the level of cultural assimilation through the name, and for a fraction of the experiments through grammatical errors that are common among native Spanish speakers using English. ${ }^{5}$ Similar to Hanson and Hawley (2011), we use quality to signal class of the tenant but add to their work by using it to further signal cultural differences between the groups. We categorize the content types of e-mail inquiries sent to landlords as High, Low type 1, and Low type 2. High-quality e-mails signal more education and do not contain mistakes using the English language. Low-quality type 1 e-mails

\footnotetext{
${ }^{5}$ We consulted with three native Spanish speakers when creating these types of errors in our correspondence, two from Colombia and one from Puerto Rico. We asked these people to write an e-mail to us inquiring about an apartment they might have an interest in and examined the content to determine common errors. It is possible that native Spanish speakers from different countries of origin make different errors. If this is true, and landlords determine origin country in addition to ethnicity from our correspondence, then our results should be viewed as discrimination based on ethnicity and country of origin.
} 
signal lower class or education by containing spelling and grammatical errors. ${ }^{6}$ Low-quality type 2 e-mails are sent by the Hispanic groups only and contain language errors. Appendix 1 shows examples of the text for all three quality categories we used to contact landlords.

We reveal tenant ethnicity to landlords through the name associated with each e-mail inquiry. Names are used to signal the tenant is either white, a Hispanic that is assimilated to American culture, or a Hispanic that is a recent immigrant. The source of first names to identify ethnicity comes from the New York City Department of Health and Human Hygiene for babies born in 1990. We use counts of babies born to non-Hispanic white mothers and Hispanic mothers to calculate the likelihood a given name is associated with each ethnicity. $L(H)$ is the likelihood a given name is from a Hispanic mother; $\mathrm{L}(\mathrm{W})$ is the likelihood a given name is from a white mother. We calculate the likelihood ratio, $\mathrm{L}(\mathrm{H}) / \mathrm{L}(\mathrm{W})$, to see how likely each name is to be considered Hispanic. There are several names that yield a ratio of infinity, ${ }^{7}$ and we consider them candidates for nonassimilated Hispanics; ratios that are finite and larger than 1 are considered for the assimilated Hispanic group. All names for white tenants have a likelihood ratio of 0 .

The source of surnames for Hispanic tenants come from the examination by Word and Perkins (1996) of 1990 Census data on the incidence of surnames among Hispanics. Word and Perkins use surnames linked to actual Census data on individual ethnicity to create classifications of how intensely last names are associated with Hispanics. All Hispanic surnames in our experiment have at least 25 occurrences in the Census and have at least $75 \%$ of households reporting Hispanic as their ethnic background; Word and Perkins label these "heavily Hispanic," and we adopt the same nomenclature here. All surnames used to represent white tenants have less than $1 \%$ of respondents reporting Hispanic as their ethnicity. To further emphasize the nonassimilated Hispanic group, we use a double surname comprised of two heavily Hispanic names, a common practice in Spanish-speaking countries, according to Midrigal et al. (2001). Table 1 shows a list of all the white, assimilated Hispanic, and nonassimilated Hispanic names in the experiment. We use only male names to ensure consistency in the within-subjects design of the experiment (each landlord receives only two emails), as we do not want to confound ethnicity and gender. ${ }^{8}$

The experiment design reflects both ethnicity differences, assimilation differences, and quality differences. Each landlord receives an e-mail from a white name with low type 1 or highquality text and also receives an e-mail from a Hispanic name (either assimilated or nonassimilated) with low type 1 , type 2 , or high-quality text. To assign landlords a particular type of audit (White-Low vs. Assimilated-Low 1, or White-Low vs. Nonassimilated-Low 2, for example), we first randomly assign them a quality type to determine if the e-mails will both be

\footnotetext{
${ }^{6}$ Our intent between high- and low-quality e-mail text is to signal the perceived quality of a prospective tenant. This may be confounded by differences in the text that convey other signals such as tenant age or seriousness of the inquiry. Using the same signal between ethnic groups maintains internal validity, but not conducting within subjects experiments across quality types limits our ability to comment on exactly how much quality of the text matters independent of an interaction with ethnicity.

${ }^{7}$ Results yield a ratio of infinity because the database does not report names for an ethnicity-gender pair with fewer than 10 babies, thus the denominator of the likelihood ratio for these names approaches zero.

${ }^{8}$ Landlords are exposed to names by the name plate in their e-mail inbox, the actual e-mail address, and an automated signature at the end of the e-mail. We construct all e-mail addresses from the same provider (Gmail) and use the same format to ensure that there are no confounding factors associated with the names signal. We follow the same format as Hanson and Hawley (2011) in creating e-mail addresses; all e-mail addresses take the form firstname.lastname. \#\#\#@gmail.com.
} 
Table 1. Names Used in Discrimination Experiment

\begin{tabular}{|c|c|c|}
\hline & Frequency of Occurrence & Percentage of E-Mails \\
\hline \multicolumn{3}{|l|}{ White names } \\
\hline Maxwell Davis & 423 & 13.78 \\
\hline Brendan Miller & 433 & 14.1 \\
\hline Ethan Olson & 405 & 13.18 \\
\hline Jack Wilson & 413 & 13.44 \\
\hline Tyler Anderson & 388 & 12.63 \\
\hline Nathan Taylor & 447 & 14.55 \\
\hline Brett Thomas & 428 & 13.93 \\
\hline Zachary Larson $^{a}$ & 49 & 1.6 \\
\hline Dylan Johnson ${ }^{a}$ & 38 & 1.24 \\
\hline Jared Moore ${ }^{\mathrm{a}}$ & 48 & 1.56 \\
\hline \multicolumn{3}{|l|}{ Assimilated Hispanic names } \\
\hline Alex Lopez & 408 & 13.28 \\
\hline Anthony Gonzalez & 413 & 13.44 \\
\hline Jonathan Santiago & 476 & 15.49 \\
\hline Christian Garcia & 409 & 13.31 \\
\hline Carlos Quintana $^{\mathrm{a}}$ & 49 & 1.6 \\
\hline \multicolumn{3}{|l|}{ Nonassimilated Hispanic names } \\
\hline Ruben Ramirez Chacon & 420 & 13.67 \\
\hline Felix Villegas Ayala & 437 & 14.22 \\
\hline Oscar Nazario Ortiz & 386 & 12.57 \\
\hline Manuel Medina Rios ${ }^{\mathrm{a}}$ & 25 & 0.81 \\
\hline Andres Lozada Alvarado & 49 & 1.6 \\
\hline
\end{tabular}

\footnotetext{
a The experiment originally began using all names; we experienced problems logging into e-mail accounts for some
} names and chose not to continue using them in the experiment after the first week of sending e-mails.

high quality or both be low quality. We then randomly assign the type of Hispanic name/ grammar combination to the audit to determine if the inquiry from a Hispanic name will be a nonassimilated or assimilated name. If the e-mail from the Hispanic name is (randomly) low quality, we randomly choose if it is to contain grammar mistakes or not (type 2). Table 2 details the possible combinations of inquiries that landlords are exposed to. ${ }^{9}$ The order in which e-mails are sent is randomly assigned to ensure that across landlords, ethnicity, and quality are not correlated with when a landlord receives an e-mail.

We assemble a group of landlords ${ }^{10}$ as test subjects using Craigslist (craigslist.org), a Web site that allows landlords to post vacancies and potential tenants to search available rental units for no monetary cost. Craigslist is organized by metropolitan area, and we choose advertisements from 21 metropolitan areas with varying levels of Hispanic population and geographic location to conduct the experiment. (Table 3 lists all metropolitan areas [MSAs] in

\footnotetext{
${ }^{9}$ The experiment does not include within ethnicity across quality variation, so we cannot isolate the effect of quality on response using within subjects tests. Our primary interest is in ethnicity, and we are able to examine differences in ethnicity across quality.

${ }^{10}$ We follow Hanson and Hawley (2011) and use landlords, rather than rental units, as the unit of observation. Hanson and Hawley find that using rental units as the unit of observations creates some suspicion by landlords who receive multiple inquiries from the same set of names.
} 
Table 2. Audit Types

White name/ high-quality e-mail

White name/ low-quality type 1 e-mail

White name/ low-quality type 1 e-mail

White name/ high-quality e-mail

White name/ low-quality type 1 e-mail

White name/ low-quality type 1 e-mail
Assimilated Hispanic name/ high-quality e-mail

Assimilated Hispanic name/ low-quality type 1 e-mail

Assimilated Hispanic name/ low-quality type 2 e-mail

Nonassimilated Hispanic name/ high-quality e-mail

Nonassimilated Hispanic name/ low-quality type 1 e-mail

Nonassimilated Hispanic name/ low-quality type 2 e-mail

We signal the race of the tenant through the name on each e-mail. Landlords are exposed to the names by the name in their e-mail inbox and the e-mail address, as well as a signature at the end of each message. The class of each e-mail is signaled by use of text in the body of the e-mail message. High-quality e-mails are written formally with good grammar and no spelling mistakes. Low-quality type 1 e-mails are less formal and contain a spelling mistake and grammatical errors. Low-quality type 2 e-mails are sent only by Hispanic auditors and contain grammatical errors common to native Spanish speakers that are not fluent in English.

the experiment. $)^{11}$ Overall, the 21 MSAs in our sample represent $35 \%$ of the national population. ${ }^{12}$ This understates the representativeness of our sample, as landlords in rural areas or smaller metro areas near major metropolitan areas post advertisements for rental units on Craigslist under the major metro markets. About $30 \%$ of units in our sample are not identified as inside of a metropolitan area as defined by the Census. The metropolitan areas in our sample represent $53 \%$ of the Hispanic population in the United States.

We draw a random sample of landlords advertising vacancies on Craigslist each week for five weeks between February 6 and March 11, 2011, to use in the experiment. To create the sample, we download all advertisements posted on Craigslist between 10 A.M. and 8 P.M. for a given city and filter the listings to ensure that we have unique landlords to contact. ${ }^{13}$ After filtering for unique landlords, we randomly select a group to contact for each city-week. The number of audits per city is highly dependent on the number of unique postings available; cities like San Antonio have very few unique postings, while New York City has the largest Craigslist housing market with many unique postings.

Using the randomly selected, unique set of landlords, we conduct the experiment by replying to landlord advertisements the day after they are posted. We use advertisements from Tuesdays and Thursdays and send inquiries on Wednesdays and Fridays between 2:30 P.M. and 5:30 P.M. We send the second inquiry to each landlord at least one hour and at most three hours after the first.

\footnotetext{
${ }^{11}$ We chose the 21 MSAs in the experiment after considering proximity to areas where Hispanics typically migrate from (Mexico and the Caribbean), current Hispanic population, and the number of unique rental postings on Craigslist. Smaller metropolitan areas with large Hispanic populations, such as El Paso, TX, and Tucson, AZ, were not included because they did not have enough unique rental postings.

12 Table 3 shows the percentage of audits represented by each MSA in our sample. The percentage of audits depends heavily on the availability of unique landlords posting units on a particular MSA's Craigslist page. Sampling only unique landlords means that the percentage of sample units in each MSA does not represent a population-weighted percentage. Using population as a base, we oversample Austin, New York, Phoenix, and San Diego, and undersample Chicago, Dallas, Houston, and Los Angeles. If we reweight our primary results to match population weights, our results are essentially identical the primary results reported in Table 5; we find that the magnitude of discrimination toward nonassimilated Hispanics increases by $0.09 \%$ of landlords.

${ }^{13}$ We filter listings automatically by e-mail address and phone number, and then inspect them manually for repeat units.
} 
Table 3. Number of Audits and Response Rate across Cities

\begin{tabular}{lrrcc}
\hline Full sample & $\begin{array}{c}\text { Number } \\
\text { of Audits }\end{array}$ & $\begin{array}{c}\text { Percentage } \\
\text { of Audits }\end{array}$ & $\begin{array}{c}\text { Overall } \\
\text { Response Rate }\end{array}$ & $\begin{array}{c}\text { Responded to at } \\
\text { Least One Inquiry }\end{array}$ \\
\hline Atlanta & 3072 & & $42.84 \%$ & $56.15 \%$ \\
Austin & 151 & $4.92 \%$ & $33.44 \%$ & $45.03 \%$ \\
Baltimore & 130 & $4.23 \%$ & $41.51 \%$ & $54.62 \%$ \\
Boston & 79 & $2.57 \%$ & $39.24 \%$ & $48.10 \%$ \\
Chicago & 110 & $3.58 \%$ & $47.28 \%$ & $66.36 \%$ \\
Dallas & 234 & $7.62 \%$ & $47.01 \%$ & $63.68 \%$ \\
Denver & 129 & $4.20 \%$ & $34.50 \%$ & $45.74 \%$ \\
Houston & 64 & $2.08 \%$ & $52.35 \%$ & $65.63 \%$ \\
Los Angeles & 109 & $3.55 \%$ & $43.12 \%$ & $55.96 \%$ \\
Miami & 235 & $7.65 \%$ & $44.26 \%$ & $55.32 \%$ \\
New York & 182 & $5.92 \%$ & $38.19 \%$ & $50.55 \%$ \\
Orlando & 642 & $20.90 \%$ & $42.06 \%$ & $56.85 \%$ \\
Phoenix & 65 & $2.12 \%$ & $31.54 \%$ & $41.54 \%$ \\
Portland & 195 & $6.35 \%$ & $46.41 \%$ & $60.51 \%$ \\
San Antonio & 63 & $2.05 \%$ & $47.62 \%$ & $63.49 \%$ \\
San Diego & 23 & $0.75 \%$ & $23.91 \%$ & $30.43 \%$ \\
San Francisco & 128 & $4.17 \%$ & $50.78 \%$ & $63.28 \%$ \\
Seattle & 143 & $4.65 \%$ & $48.25 \%$ & $59.44 \%$ \\
St. Louis & 58 & $1.89 \%$ & $62.07 \%$ & $74.14 \%$ \\
Tampa & 99 & $3.22 \%$ & $32.83 \%$ & $47.47 \%$ \\
Washington, DC & 60 & $1.95 \%$ & $45.00 \%$ & $53.33 \%$ \\
\hline \multicolumn{1}{c}{ Ta } & 173 & $5.63 \%$ & $42.77 \%$ & $56.07 \%$ \\
\hline
\end{tabular}

The experiment ran for five weeks with two rounds of sending e-mails per week. During the first three weeks 252 audits were sent per round. During the last two weeks of sending the audits sent per round were increased to approximately 350 audits. The number of audits per city depended on the relative availability of unique postings.

\section{Validating Assimilation Signals}

The assimilated and nonassimilated Hispanic names in our experiment are intended to provide a signal to landlords about the level of cultural assimilation of a potential tenant. We also use the text of our e-mail inquiries to further enforce the assimilation signal. Birth certificate data support the claim that the names in the experiment are significantly more likely to be associated with Hispanics; however, using more heavily Hispanic names and double last names does not necessarily signal cultural assimilation. In addition, although we assume that the text of our e-mails adds to the cultural assimilation signal, there is no preexisting evidence to support these claims.

To explore the notion that the names in our survey signal cultural assimilation and the text of our inquiries reinforces this signal, we conduct a survey. The survey, detailed in Appendix 2, consists of two sections. The first section asks respondents to indicate if the names in our survey convey any information about a person's familiarity with American customs and culture. Respondents were asked to fill in boxes indicating if the name indicated a person was "Very Unfamiliar," "Unfamiliar," "Neutral," "Familiar," or "Very Familiar" with American customs and culture. The second section asked respondents to indicate if the author of our e-mail texts indicated familiarity with American customs and culture (using the same scale as with names).

The survey results show strong support for our assumption that the names in our experiment convey different levels of cultural assimilation. Appendix Table 1 shows a summary 
of the results by name. For all the white names in the survey, the majority of respondents indicated that the names conveyed a person either "familiar" or "very familiar" with American customs and culture. For the assimilated Hispanic group, the percentage of respondents indicating that names conveyed a person who was "very familiar" with American customs and culture slipped substantially, but most respondents indicated these names conveyed someone either "familiar" or "neutral" with American customs and culture. ${ }^{14}$ The nonassimilated Hispanic group shows a substantial drop from the assimilated group, where the majority of respondents indicate these names indicate a person is either "unfamiliar" or "very unfamiliar" with American customs and culture.

To further examine the statistical significance of the survey results, we estimate linear probability and Probit regressions with the data. These regressions use a dependent variable equal to one if the respondent indicated a particular name was either familiar or very familiar with American customs and culture, and equal to zero if the respondent indicated the name was either unfamiliar or very unfamiliar with American customs and culture (neutral responses are left blank in the regression, so they do not add to the marginal probability of names and text being perceived as either familiar or unfamiliar). We estimate the marginal probability of indicating a name was familiar or very familiar with American customs and culture by creating dummy variables for each name (excluding the name Brendan Miller) to use as independent variables. We estimate these regressions with and without respondent fixed effects.

All of these models, linear probability or Probit, with or without respondent fixed effects, show strong support for the idea that the names in our survey indicate cultural assimilation. All of the white names, with the exception of Jared Moore in the Probit specifications, are statistically indistinguishable from each other. All of the assimilated Hispanic names show that respondents are $20-60 \%$ less likely (depending on the model) to indicate they are familiar or very familiar with American customs and culture, with the exception that Carlos Quintana appears to be interpreted more like a nonassimilated name. The nonassimilated Hispanic names show a larger marginal effect-that respondents are 60-80\% less likely (depending on the model) to indicate they convey familiarity with American customs and culture relative to the reference white name. ${ }^{15}$ Appendix Table 2 details these results.

The survey also shows strong support for the assumption that e-mail text in our experiment helps to convey a level of cultural assimilation. Appendix Table 3 details the results of the survey for e-mail texts. The vast majority of respondents indicated that the high-quality e-mail text conveyed a person was either "familiar" or "very familiar" with American customs and culture. For the low-quality type 1 e-mail, the survey results show an approximately equal proportion of respondents thought this text conveyed familiarity as did unfamiliarity, with many respondents choosing "neutral." A large majority of respondents indicated that the

\footnotetext{
${ }^{14}$ Interestingly, our survey seems to suggest the name Carlos Quintana as a possible outlier. This was an assimilated name according to our classification but was perceived to be similar to the nonassimilated group of Hispanic names. Our experiment contained relatively few observations using Carlos Quintana, and dropping them does not change our primary results. These results also do not change if we reclassify Carlos Quintana as a nonassimilated name.

${ }^{15}$ To explore how the choice of coding neutral responses affects our interpretation of the survey data, we recoded neutral responses as either a zero (adding to the marginal probability of unfamiliar with American customs and culture) or one (adding to the marginal probability of being familiar). In either case, the results are extremely similar in spirit to Appendix Tables 2 and 4. Recoding neutral responses as familiar makes the coefficient estimates slightly less negative for assimilated and nonassimilated names and slightly lowers the difference in estimated magnitudes. Recoding neutral responses as unfamiliar does the opposite, but to a lesser degree, and pushes some of the white names to test statistically different than the baseline.
} 
low-quality type 2 e-mail, which was used only with the nonassimilated names, conveyed a person as either "unfamiliar" or "very unfamiliar" with American customs and culture.

As with the survey data on names, we estimate linear probability and Probit regressions to explore the survey data for e-mail texts. The dependent variable is equal to one if respondents indicated the e-mail signaled the author was either familiar or very familiar with American customs and culture, and zero if they indicated they were unfamiliar or very unfamiliar. Each regression includes a dummy variable equal to one if the response was for the low-quality type 1 , and zero otherwise, and another dummy variable equal to one if the response was for the low-quality type 2 (signaling nonassimilated), and zero otherwise. The reference group is the high-quality e-mail. We estimate these regressions with and without respondent fixed effects.

These models all show strong support for the assumption that the text of our e-mails indicate a level of cultural assimilation. Low-quality e-mails were 46-64\% less likely to be perceived as familiar or very familiar with American customs and culture than the high-quality e-mails. The low-quality type 2 e-mails were $84-93 \%$ less likely to be perceived as familiar or very familiar with American customs and culture than the high-quality e-mails. Although we did not expect that the low-quality type 1 e-mails indicate a lower level of cultural assimilation, it is clear that this is not as strong of a signal as the low-quality type 2 e-mails.

\section{Sample Characteristics}

Our experiment includes 3072 audits or 6144 e-mails across 21 metropolitan areas. The overall response rate is $42.84 \%$ with $56.15 \%$ of landlords responding to at least one of the inquiries. Table 3 provides a breakdown of the response rates across metropolitan areas. The number of audits per area ranges from 23 in San Antonio to 642 in New York City with an average of 146 audits per area. Overall, the response rate varies from $23.91 \%$ in San Antonio to $62.07 \%$ in Seattle.

We use advertised characteristics of housing units to describe the sample of rental units. Using a scraping technique, we obtain characteristics for over $98 \%$ of the sample using the description provided by the landlord that posted the unit on Craigslist. Unit-level characteristics include number of bedrooms and bathrooms, unit type (single-family home, duplex, townhouse, condominium, apartment, or shared room), monthly rent, square footage, whether the unit was posted by a company or not, and whether the landlord advertised a discount or not.

In the full sample, $68 \%$ of units are apartments and $16 \%$ are single-family homes, with the remainder described as a shared room, duplex, condominium, or townhouse. Shared rooms are the least common unit type at just under $1 \%$ of the sample. The average monthly rent is $\$ 1516$ with an average of 1.92 bedrooms, 1.5 bathrooms, and 1163 square feet. A company listed approximately $75 \%$ of all postings, and just over $10 \%$ of units advertised a discount. Table 4 shows all sample characteristics for the full sample and for each metropolitan area.

Not surprisingly, there are stark differences between the average housing unit characteristics across the metropolitan areas in our sample. All areas besides San Francisco and Miami have greater than $50 \%$ apartments in the sample, while New York, Boston, Chicago, and San Antonio have a greater than $80 \%$ share. St. Louis has by far the greatest share of single-family homes for rent with over $43 \%$ of units, while Baltimore, Boston, New 


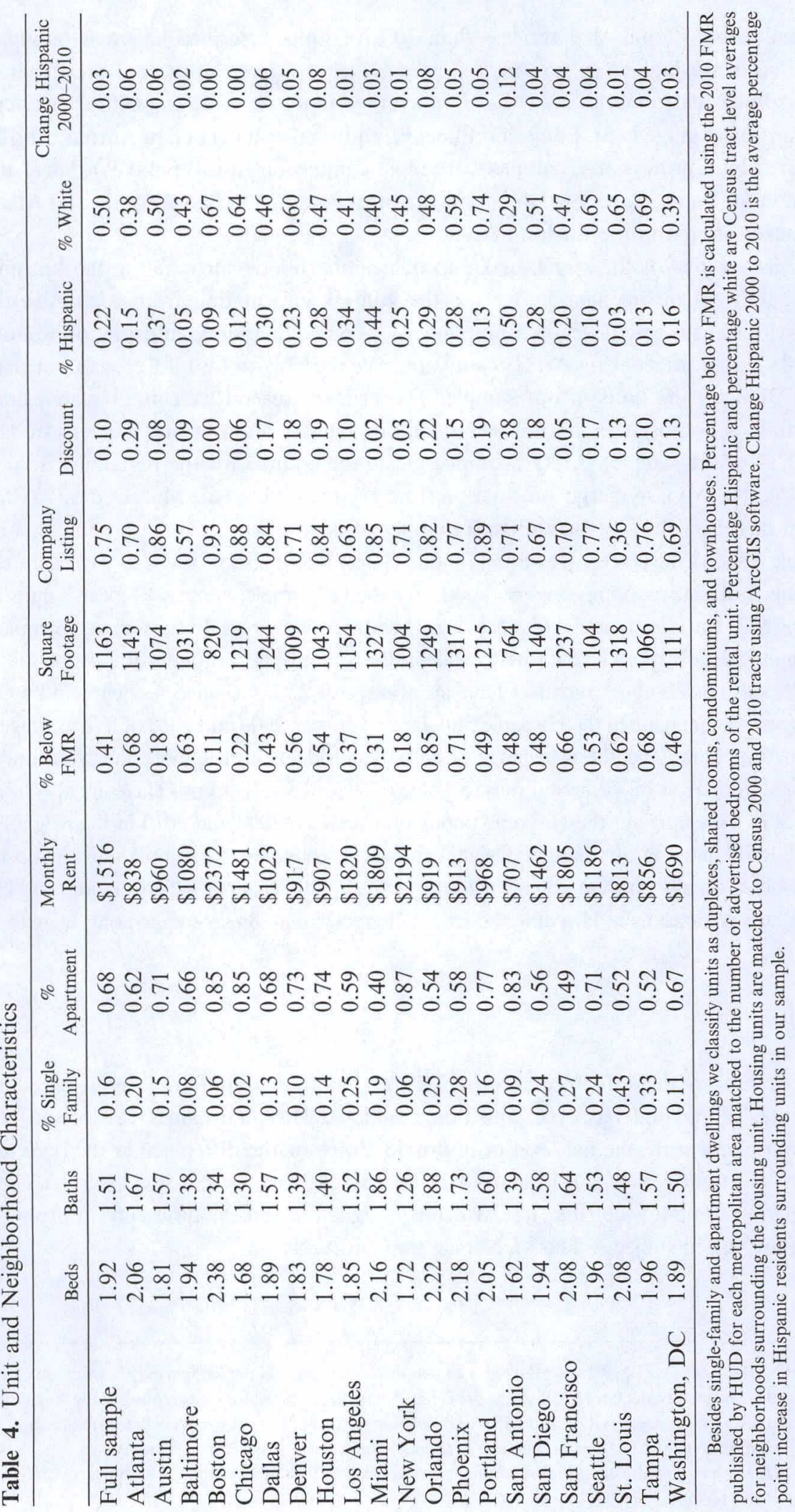


York, and San Antonio all have less than $10 \%$ of units described as single-family homes. Average rents are highest in Boston, followed closely by New York, and are lowest in San Antonio. Most metropolitan areas have an average unit size close to 1000 square feet, with larger units on average in St. Louis and Phoenix, and smaller units in San Antonio and Boston. Units listed by a professional company are most common in Boston and Portland, and least common in St. Louis. Advertised discounts are most common in San Antonio and Atlanta but almost never offered in the Boston market.

To get an idea of the ethnic make-up of neighborhoods surrounding the housing units available for rent in our sample, we use the address information from each advertisement merged with Census tract level data on ethnicity. We match address information to both 2000 and 2010 Census tracts using ArcGIS software. We are able to find a Census tract match for $70 \%$, or 2168 , of the units in our sample. The primary reason we cannot match units to a Census tract is because they do not include address information, which happens in 840 cases (about $27 \%$ of the sample). ArcGIS cannot locate the address for the remaining nonmatched units (64 units). We also merge information from HUD on the "Fair Market Rent" (FMR) for each unit based on the city location and number of bedrooms. ${ }^{16}$

Table 4 shows the percentage of units in our sample renting below FMR as well as the ethnicity of residents in the surrounding neighborhood. For the full sample, about $40 \%$ of our units rent for less than FMR, with substantial differences across metropolitan areas. In the Orlando sample, nearly $85 \%$ of units are advertised below FMR, while in Boston only $11 \%$ of units are. In the full sample, neighborhoods surrounding our units have an average of $22 \%$ Hispanic residents and $50 \%$ white residents, with an increase in the Hispanic population between 2000 and 2010 of 3 percentage points. Across metropolitan areas the percentage of Hispanic residents in neighborhoods surrounding our units ranges from a low of $3 \%$ in St. Louis to a high of 50\% in San Antonio. There are also substantial differences in the change in the Hispanic population between 2000 and 2010 in the neighborhoods surrounding the units in our sample. Neighborhoods surrounding the sample units in Boston and Chicago show virtually no change in Hispanic residents, ${ }^{17}$ while San Antonio, Orlando, and Houston show the largest increases in Hispanic residents (all greater than 8 percentage point increase).

\section{Results}

Table 5 shows the difference in response from landlords to inquiries about rental housing using white, assimilated Hispanic, and nonassimilated Hispanic names. All of our tests for discrimination measure the net level of discrimination - or the difference in the percentage of landlords that treat whites and Hispanics differently. We test for statistical significance of net discrimination using a McNemar test, which is designed to test the difference in proportion of response for paired subjects. The McNemar test statistic is

$$
\chi^{2}=\left(N_{\text {Only White }}-N_{\text {Only Hispanic }}\right)^{2} /\left(N_{\text {Only White }}+N_{\text {Only Hispanic }}\right),
$$

\footnotetext{
${ }^{16}$ We use the 2011 fiscal year FMR from HUD as a way to show what units in our sample may be attainable for tenants with low incomes. HUD calculates FMR as the gross rent (including utilities) for units renting at the 40th percentile in the distribution of each city. HUD uses FMRs to determine payments for housing voucher programs and rents for Section 8 contracts, as well as for a benchmark in several other housing assistance programs.

${ }^{17}$ Chicago actually has a small negative average change of less than $1 / 10$ th of $1 \%$, while Boston has an extremely small average positive change.
} 
Table 5. Landlord Response and Tests for Discrimination

\begin{tabular}{lccccc}
\hline & Reply Neither & Reply Both & White Only & Hispanic Only & $\mathrm{H}_{\mathrm{O}}: \mathrm{R}_{\mathrm{W}}-\mathrm{R}_{\mathrm{H}}=0$ \\
\hline Audits with & & & & & \\
assimilated & & & & & \\
Hispanic names & & & & & \\
All audits & $42.79 \%$ & $31.23 \%$ & $12.08 \%$ & $13.90 \%$ & $-1.82 \%$ \\
& {$[751]$} & {$[548]$} & {$[212]$} & {$[244]$} & $p=0.1465$ \\
High quality & $42.33 \%$ & $28.84 \%$ & $15.01 \%$ & $13.83 \%$ & $1.18 \%$ \\
& {$[251]$} & {$[171]$} & {$[89]$} & {$[82]$} & $p=0.6465$ \\
Low quality (1) & $43.27 \%$ & $30.55 \%$ & $11.45 \%$ & $14.73 \%$ & $-3.28 \%$ \\
& {$[238]$} & {$[168]$} & {$[63]$} & {$[81]$} & $p=0.1563$ \\
Low quality (2) & $42.81 \%$ & $34.15 \%$ & $9.80 \%$ & $13.24 \%$ & $-3.44 \% *$ \\
& {$[262]$} & {$[209]$} & {$[60]$} & {$[81]$} & $p=0.0918$ \\
Audits with & & & & & \\
nonassimilated & & & & $15 \%$ & \\
Hispanic names & & & & & \\
All audits & $45.25 \%$ & $27.26 \%$ & $15.19 \%$ & $12.30 \%$ & $2.89 \% *$ \\
& {$[596]$} & {$[359]$} & {$[200]$} & {$[162]$} & $p=0.0517$ \\
High quality & $44.06 \%$ & $27.63 \%$ & $13.93 \%$ & $14.38 \%$ & $-0.45 \%$ \\
Low quality (1) & {$[193]$} & {$[121]$} & {$[61]$} & {$[63]$} & $p=0.9285$ \\
& $46.15 \%$ & $28.67 \%$ & $14.22 \%$ & $10.96 \%$ & $3.26 \%$ \\
Low quality (2) & {$[198]$} & {$[123]$} & {$[61]$} & {$[47]$} & $p=0.2108$ \\
& $45.56 \%$ & $25.56 \%$ & $17.33 \%$ & $11.56 \%$ & $5.77 \% * *$ \\
\hline & {$[205]$} & {$[115]$} & {$[78]$} & {$[52]$} & $p=0.0279$ \\
\hline
\end{tabular}

Number of observations in square brackets reflect the number of landlords that respond to an inquiry from neither, both, or only one of the groups. All tests of statistical significance are from McNemar paired difference in proportions tests, which follow a chi-square distribution. Assimilated Hispanics have a first name with $1<\mathrm{L}(\mathrm{W}) / \mathrm{L}(\mathrm{H})<\infty$, and a single Hispanic surname. Nonassimilated Hispanics have a first name with $\mathrm{L}(\mathrm{H}) / \mathrm{L}(\mathrm{W})=\infty$ and two Hispanic surnames. High-quality e-mails are written formally with good grammar and no spelling mistakes. Low-quality type 1 e-mails are less formal and contain a spelling mistake and grammatical errors. Low-quality type 2 e-mails are sent only by Hispanic auditors and contain grammatical errors common to native Spanish speakers that are not fluent in English; for these audits white tenants always send low-quality type 1 e-mails.

* Significant at the $10 \%$ level.

** Significant at the $5 \%$ level.

where $N$ is the number of landlords. The test statistic has a chi-square distribution, and we calculate $p$ values accordingly.

The results in Table 5 show fairly strong evidence that landlords discriminate against nonassimilated Hispanics searching for rental housing. When combining all audit types, we find that on net, about $2.9 \%$ of landlords discriminate by not responding to nonassimilated Hispanics, statistically significant at the $5 \%$ level. We also find that discrimination against the nonassimilated Hispanic group is largely driven by differences between low-quality white e-mails and low-quality e-mails from Hispanics that contain grammatical errors common to native Spanish speakers (type 2). These audits show about $5.8 \%$ of landlords practicing unequal treatment, statistically significant at the 5\% level. We do not find any other statistically significant difference between the nonassimilated group and whites, with only small differences in magnitude when both inquiries are of high quality. ${ }^{18}$ The magnitude of the difference when both groups are low-quality type 1 is larger, about $3.2 \%$ of landlords, but not statistically

\footnotetext{
${ }^{18}$ We did not design the within subjects experiment to test for quality independent of ethnicity, so we cannot definitively say how much of the differences are due to e-mail text and how much are due to the interaction with the text and the names in our experiment.
} 
Table 6. Landlord Response and Tests for Discrimination by Region

\begin{tabular}{lccccc}
\hline & Reply Neither & Reply Both & White Only & Hispanic Only & $\mathrm{H}_{\mathrm{o}}: \mathrm{R}_{\mathrm{W}}-\mathrm{R}_{\mathrm{H}}=0$ \\
\hline New York & $43.15 \%$ & $27.26 \%$ & $14.64 \%$ & $14.95 \%$ & $-0.31 \%$ \\
& {$[277]^{\circ}$} & {$[175]$} & {$[94]$} & {$[96]$} & $p=0.9422$ \\
Northeast/Midwest & $41.87 \%$ & $28.06 \%$ & $14.82 \%$ & $15.25 \%$ & $-0.43 \%$ \\
& {$[291]$} & {$[195]$} & {$[103]$} & {$[106]$} & $p=0.8900$ \\
Southeast & $52.18 \%$ & $25.33 \%$ & $10.26 \%$ & $12.23 \%$ & $-1.97 \%$ \\
& {$[239]$} & {$[116]$} & {$[47]$} & {$[56]$} & $p=0.4307$ \\
Southern California & $41.87 \%$ & $34.99 \%$ & $9.64 \%$ & $13.50 \%$ & $-3.86 \%$ \\
& {$[152]$} & {$[127]$} & {$[35]$} & {$[49]$} & $p=0.1557$ \\
Northwest & $35.98 \%$ & $38.72 \%$ & $13.72 \%$ & $11.59 \%$ & $2.13 \%$ \\
Southwest & {$[118]$} & {$[127]$} & {$[45]$} & {$[38]$} & $p=0.5104$ \\
& $46.08 \%$ & $28.50 \%$ & $15.02 \%$ & $10.41 \%$ & $4.61 \% * *$ \\
& {$[270]$} & {$[167]$} & {$[88]$} & {$[61]$} & $p=0.0328$ \\
\hline
\end{tabular}

Number of observations in square brackets reflect the number of landlords that respond to an inquiry from neither, both, or only one of the groups. All tests of statistical significance are from McNemar paired difference in proportions tests, which follow a chi-square distribution. Northeast/Midwest region includes Boston, Washington, DC, Baltimore, Chicago, and St. Louis. Southeast region includes Atlanta, Orlando, Tampa, and Miami. Southern California includes San Diego and Los Angeles. Northwest region includes San Francisco, Portland, Seattle, and Denver. Southwest region includes Houston, Austin, Dallas, San Antonio, and Phoenix.

** Significant at the $5 \%$ level.

meaningful. The results in Table 5 show no evidence of discrimination against Hispanics with assimilated names; in fact, they have a marginally statistically significant higher response rate than whites when both use low-quality e-mails (with Hispanics sending low-quality type 2).

Table 6 shows results of tests for discrimination across different regions of the United States. There are vast differences in both the number of Hispanic residents in different parts of the United States as well as the composition of Hispanics. For example, the majority of Hispanics (51\%) in Miami report their country of origin to be Cuba, while Mexicans are the primary Hispanic group in Los Angeles (Lopez and Dockterman 2011). Because Hispanics are a heterogeneous group across regions, we examine possible differences in discrimination by region. ${ }^{19}$ Results of landlord response by region show that the only statistically significant discrimination we find against Hispanics occurs in the Southwestern United States, which includes Houston, Austin, Dallas, San Antonio, and Phoenix. We find net discrimination of about $4.6 \%$ of landlords in the Southwest region, statistically significant at the $5 \%$ level. No other region shows statistically significant levels of discrimination in our sample, and in fact in Southern California Hispanics show a positive differential, although this is not statistically meaningful. Interestingly, Hispanics in Southern California (Los Angeles) and Texas (Houston and San Antonio) are both overwhelmingly from Mexico, so it appears it is not the origin of Hispanics, but rather white attitudes toward them across regions that is driving discrimination.

\section{Results by Neighborhood and Unit Characteristics}

Using the information on unit characteristics from the posted advertisements and the neighborhood ethnicity from our Census tract match, we can test for differences in

\footnotetext{
${ }^{19}$ Our sample size is not large enough to test for discrimination at the city level in most cases besides New York; it is also not large enough to divide groups between assimilated and nonassimilated in most cases at the region level, so we report results of combined tests only.
} 
Table 7. Landlord Response and Tests for Discrimination by Neighborhood Ethnicity

\begin{tabular}{cccccr}
\hline & Reply Neither & Reply Both & White Only & Hispanic Only & $\mathrm{H}_{\mathrm{o}}: \mathrm{R}_{\mathrm{W}}-\mathrm{R}_{\mathrm{H}}=0$ \\
\hline Assimilated Hispanics & & & & & \\
1st quartile $(<7.5 \%$ & $39.26 \%$ & $31.60 \%$ & $11.96 \%$ & $17.18 \%$ & $-5.21 \%$ \\
Hispanic) & {$[128]$} & {$[103]$} & {$[39]$} & {$[56]$} & $p=0.1002$ \\
2nd quartile $(7.6-15 \%$ & $43.47 \%$ & $31.00 \%$ & $12.46 \%$ & $13.07 \%$ & $-0.61 \%$ \\
Hispanic) & {$[143]$} & {$[102]$} & {$[41]$} & {$[43]$} & $p=0.9132$ \\
3rd quartile $(15.1-31.7 \%$ & $40.72 \%$ & $32.25 \%$ & $12.38 \%$ & $14.66 \%$ & $-2.28 \%$ \\
Hispanic) & {$[125]$} & {$[99]$} & {$[38]$} & {$[45]$} & $p=0.5104$ \\
4th quartile $(>31.8 \%$ & $43.37 \%$ & $34.63 \%$ & $9.71 \%$ & $12.30 \%$ & $-2.59 \%$ \\
Hispanic) & {$[134]$} & {$[107]$} & {$[30]$} & {$[38]$} & $p=0.3961$ \\
Nonassimilated Hispanics & & & & & \\
1st quartile $(<7.5 \%$ & $46.89 \%$ & $25.36 \%$ & $14.83 \%$ & $12.92 \%$ & $1.91 \%$ \\
Hispanic) & {$[98]$} & {$[53]$} & {$[31]$} & {$[27]$} & $p=0.6940$ \\
2nd quartile $(7.6-15 \%$ & $46.30 \%$ & $27.78 \%$ & $17.13 \%$ & $8.80 \%$ & $8.33 \% * *$ \\
Hispanic) & {$[100]$} & {$[60]$} & {$[37]$} & {$[19]$} & $p=0.0222$ \\
3rd quartile $(15.1-31.7 \%$ & $42.42 \%$ & $29.44 \%$ & $16.45 \%$ & $11.69 \%$ & $4.76 \%$ \\
Hispanic) & {$[98]$} & {$[68]$} & {$[38]$} & {$[27]$} & $p=0.2145$ \\
4th quartile $(>31.8 \%$ & $42.86 \%$ & $30.74 \%$ & $13.85 \%$ & $12.55 \%$ & $1.30 \%$ \\
Hispanic) & {$[99]$} & {$[71]$} & {$[32]$} & {$[29]$} & $p=0.7982$ \\
\hline
\end{tabular}

Number of observations in square brackets reflect the number of landlords that respond to an inquiry from neither, both, or only one of the groups. All tests of statistical significance are from McNemar paired difference in proportions tests, which follow a chi-square distribution. Neighborhood percentage Hispanic is from the 2010 Census tract level data. Unit Census tracts are identified using ArcGIS software.

* Significant at the $10 \%$ level.

** Significant at the $5 \%$ level.

discrimination across characteristics and examine how they may impact discrimination. Looking across characteristics and neighborhood ethnicity may offer some insight as to why discrimination occurs. Card, Mas, and Rothstein (2008) suggest that the share of minorities in an area has a substantial effect on out-migration of white residents. They suggest that neighborhoods exhibit tipping behavior when the minority share becomes too high-when this happens nearly all white residents leave the neighborhood. If landlords want to prevent neighborhood tipping, they may be more likely to discriminate in neighborhoods where the share of nonwhites is near a tipping point. ${ }^{20} \mathrm{We}$ also expect that landlords are simply less likely to discriminate against Hispanics in areas where they are more concentrated as landlords may perceive these areas to be suitable for Hispanics to live. This is the perceived customer prejudice hypothesis highlighted by Yinger (1986).

Table 7 shows discrimination tests by the percentage of Hispanic residents in neighborhoods surrounding housing units in the sample. We find that among the nonassimilated group, discrimination occurs only in the second quartile of the distribution (the second least amount of Hispanics). These results show that more than $8.3 \%$ of landlords discriminate against Hispanics on net, statistically significant at the 5\% level. For the assimilated Hispanic group, we actually find discrimination against whites in neighborhoods

\footnotetext{
${ }^{20}$ Card Mas and Rothstein suggest a tipping point between 5\% and 20\% minority share, depending on the city. Our data become thin if we try to use these breaks, so we instead separate out data into quartiles to test for differences across ethnic composition of neighborhoods.
} 
Table 8. Landlord Response and Tests for Discrimination by Change in Neighborhood Ethnicity

\begin{tabular}{cccccc}
\hline & Reply Neither & Reply Both & White Only & Hispanic Only & $\mathrm{H}_{\mathrm{o}}: \mathrm{R}_{\mathrm{W}}-\mathrm{R}_{\mathrm{H}}=0$ \\
\hline Assimilated Hispanics & & & & & \\
1st quartile $(<0.2 \%$ & $39.07 \%$ & $33.44 \%$ & $9.93 \%$ & $17.55 \%$ & $-7.62 \% * *$ \\
increase) & {$[118]$} & {$[101]$} & {$[30]$} & {$[53]$} & $p=0.0152$ \\
2nd quartile $(0.3-2.2 \%$ & $38.63 \%$ & $33.02 \%$ & $13.08 \%$ & $15.26 \%$ & $-2.18 \%$ \\
increase) & {$[124]$} & {$[106]$} & {$[42]$} & {$[49]$} & $p=0.5296$ \\
3rd quartile $(2.3-6.2 \%$ & $45.05 \%$ & $29.35 \%$ & $12.97 \%$ & $12.63 \%$ & $0.34 \%$ \\
increase ) & {$[132]$} & {$[86]$} & {$[38]$} & {$[37]$} & $p=1$ \\
4th quartile $(>6.3 \%$ & $41.93 \%$ & $35.09 \%$ & $9.63 \%$ & $13.35 \%$ & $-3.73 \%$ \\
increase) & {$[135]$} & {$[113]$} & {$[31]$} & {$[43]$} & $p=0.2007$ \\
Nonassimilated Hispanics & & & & & \\
1st quartile $(<0.2 \%$ & $44.93 \%$ & $23.19 \%$ & $16.91 \%$ & $14.98 \%$ & $1.93 \%$ \\
increase) & {$[93]$} & {$[48]$} & {$[35]$} & {$[31]$} & $p=0.7122$ \\
2nd quartile $(0.3-2.2 \%$ & $45.37 \%$ & $27.80 \%$ & $17.07 \%$ & $9.76 \%$ & $7.32 \% * *$ \\
point increase) & {$[93]$} & {$[57]$} & {$[35]$} & {$[20]$} & $p=0.0581$ \\
3rd quartile $(2.3-6.2 \%$ & $45.61 \%$ & $28.95 \%$ & $14.04 \%$ & $11.40 \%$ & \\
increase) & {$[104]$} & {$[66]$} & {$[32]$} & {$[26]$} & $p=0.53 \%$ \\
4th quartile $(>6.3 \%$ & $39.91 \%$ & $35.68 \%$ & $14.08 \%$ & $10.33 \%$ & \\
increase) & {$[85]$} & {$[76]$} & {$[30]$} & {$[22]$} & $p=0.3317$ \\
\hline
\end{tabular}

Number of observations in brackets reflect the number of landlords that respond to an inquiry from neither, both, or only one of the groups. All tests of statistical significance are from McNemar paired difference in proportions tests, which follow a chi-square distribution. Neighborhood percentage Hispanic is from the 2000 and 2010 Census tract level data. Unit Census tracts are identified using ArcGIS software.

** Significant at the $5 \%$ level.

with the fewest Hispanic residents. The combination of these results suggests that landlords may perceive that residents prefer some integration, but not too much (they start to discriminate as the percentage of Hispanics becomes too large). It also suggests that they may prefer the integration to come from assimilated Hispanics and thus may be sensitive to keeping out recent immigrants. Table 8 shows discrimination test results by the change in Hispanic residents at the neighborhood level between 2000 and 2010. These results are quite similar to the percentage of Hispanics in the neighborhood results, as we find areas that had the fewest amount of Hispanics entering throughout the decade actually practice discrimination against white residents, while those with the second highest Hispanic inflow are the biggest discriminators against Hispanics.

To further examine discrimination by characteristics of the surrounding neighborhood, we test for discrimination by the percentage of white residents in the neighborhood. If landlords perceive some preference for integration, we would expect to see less (or nonexistent) discrimination in neighborhoods with the highest concentration of whites; however, if landlords want to prevent tipping or perceive tenant preferences for segregation we would find more discrimination in the neighborhoods with the highest concentration of white residents. The results presented in Table 9 show that we find discrimination against nonassimilated Hispanics at both extremes of the white resident distribution. Neighborhoods in the top quartile have the strongest discrimination ( $7.3 \%$ of landlords), whereas those in the bottom quartile show weaker evidence of discrimination ( $6.2 \%$ of landlords, statistically significant at the $10 \%$ level). In our sample, neighborhoods with few whites are not necessarily where Hispanics live, so 
Table 9. Landlord Response and Tests for Discrimination by Percentage of White Residents

\begin{tabular}{cccccc}
\hline & Reply Neither & Reply Both & White Only & Hispanic Only & $\mathrm{H}_{\mathrm{O}}: \mathrm{R}_{\mathrm{W}}-\mathrm{R}_{\mathrm{H}}=0$ \\
\hline Assimilated Hispanics & & & & & \\
1st quartile $(<28 \%$ & $45.98 \%$ & $28.30 \%$ & $9.65 \%$ & $16.08 \%$ & $-6.43 \% * *$ \\
white) & {$[143]$} & {$[88]$} & {$[30]$} & {$[50]$} & $p=0.0330$ \\
2nd quartile (28-55\% & $42.99 \%$ & $34.08 \%$ & $9.55 \%$ & $13.38 \%$ & $-3.82 \%$ \\
white) & {$[135]$} & {$[107]$} & {$[30]$} & {$[42]$} & $p=0.1945$ \\
3rd quartile (56-72\% & $41.67 \%$ & $32.41 \%$ & $12.96 \%$ & $12.96 \%$ & $0.00 \%$ \\
white) & {$[135]$} & {$[105]$} & {$[42]$} & {$[42]$} & $p=1.0000$ \\
4th quartile (>72\% & $36.34 \%$ & $34.47 \%$ & $14.29 \%$ & $14.91 \%$ & $-0.62 \%$ \\
white) & {$[117]$} & {$[111]$} & {$[46]$} & {$[48]$} & $p=0.9179$ \\
Nonassimilated Hispanics & & & & & \\
1st quartile $(<28 \%$ & $50.22 \%$ & $23.77 \%$ & $16.14 \%$ & $9.87 \%$ & $6.28 \% *$ \\
white) & {$[112]$} & {$[53]$} & {$[36]$} & {$[22]$} & $p=0.0869$ \\
2nd quartile (28-55\% & $39.30 \%$ & $30.57 \%$ & $15.28 \%$ & $14.85 \%$ & $0.44 \%$ \\
white) & {$[90]$} & {$[70]$} & {$[35]$} & {$[34]$} & $p=1.0000$ \\
3rd quartile (56-72\% & $43.98 \%$ & $33.33 \%$ & $12.50 \%$ & $10.19 \%$ & $2.31 \%$ \\
white) & {$[95]$} & {$[72]$} & {$[27]$} & {$[22]$} & $p=0.5682$ \\
4th quartile $(>72 \%$ & $44.75 \%$ & $26.03 \%$ & $18.26 \%$ & $10.96 \%$ & \\
white) & {$[98]$} & {$[57]$} & {$[40]$} & {$[24]$} & $p=0.0599$ \\
\hline
\end{tabular}

Number of observations in square brackets reflect the number of landlords that respond to an inquiry from neither, both, or only one of the groups. All tests of statistical significance are from McNemar paired difference in proportions tests, which follow a chi-square distribution. Neighborhood percentage white is from the 2010 Census tract level data. Unit Census tracts are identified using ArcGIS software.

* Significant at the $10 \%$ level.

** Significant at the $5 \%$ level.

discrimination at this part of the distribution could be where other groups live. We should also note that we do find substantial discrimination against whites compared to assimilated Hispanics in neighborhoods with the fewest whites.

Discrimination is also sensitive to the type of unit advertised and the rental price with respect to the FMR in the area. Table 10 shows tests of discrimination for the sample broken down by type of unit (single family, apartment), if a company made the listing, and if the rent is below FMR. We find no difference for the assimilated Hispanics, but large differences for the nonassimilated group. We find statistically significant discrimination against nonassimilated Hispanics in apartment buildings, but not in single-family home rentals (although the magnitude favors whites). Somewhat surprisingly, we find discrimination against nonassimilated Hispanics for units that rent for less than the FMR in the area. One possible explanation for this is landlords in this price range may carry more stereotypes against minority renters if they deal with them more often.

\section{Results for Response Content}

Our primary evidence shows that landlords discriminate against nonassimilated Hispanics by not responding to e-mail inquiries about rental housing. It is also possible that they respond to both groups, but still do not treat them equally. Measuring discrimination as the difference between response and nonresponse misses any unequal treatment from landlords that reply to both inquiries, but with a different response. Using only response/nonresponse does not measure the difference between landlords that respond negatively to whites and positively to 
Table 10. Landlord Response and Tests for Discrimination by Unit Characteristics

\begin{tabular}{lccccc}
\hline & Reply Neither & Reply Both & White Only & Hispanic Only & $\mathrm{H}_{\mathrm{o}}: \mathrm{R}_{\mathrm{W}}-\mathrm{R}_{\mathrm{H}}=0$ \\
\hline Assimilated Hispanics & & & & & \\
Single-family homes & $40.00 \%$ & $33.33 \%$ & $10.83 \%$ & $15.83 \%$ & $-5.00 \%$ \\
& {$[96]$} & {$[80]$} & {$[26]$} & {$[38]$} & $p=0.1686$ \\
Apartments & $43.18 \%$ & $31.01 \%$ & $11.88 \%$ & $13.93 \%$ & $-2.04 \%$ \\
& {$[465]$} & {$[334]$} & {$[128]$} & {$[150]$} & $p=0.2078$ \\
Company listed & $44.11 \%$ & $29.04 \%$ & $12.54 \%$ & $14.31 \%$ & $-1.77 \%$ \\
& {$[524]$} & {$[345]$} & {$[149]$} & {$[170]$} & $p=0.2628$ \\
Rent below HUD fair & $46.47 \%$ & $31.21 \%$ & $10.31 \%$ & $12.01 \%$ & $-1.69 \%$ \\
market rate & {$[329]$} & {$[221]$} & {$[73]$} & {$[85]$} & $p=0.2710$ \\
Nonassimilated Hispanics & & & & & \\
Single-family homes & $38.19 \%$ & $31.66 \%$ & $17.59 \%$ & $12.56 \%$ & $5.03 \%$ \\
& {$[76]$} & {$[63]$} & {$[35]$} & {$[25]$} & $p=0.2451$ \\
Apartments & $46.72 \%$ & $27.40 \%$ & $14.90 \%$ & $10.98 \%$ & $3.91 \% * *$ \\
& {$[370]$} & {$[217]$} & {$[118]$} & {$[87]$} & $p=0.0359$ \\
Company listed & $45.30 \%$ & $25.67 \%$ & $15.56 \%$ & $13.47 \%$ & $2.09 \%$ \\
& {$[390]$} & {$[221]$} & {$[134]$} & {$[116]$} & $p=0.2823$ \\
Rent below HUD fair & $48.27 \%$ & $27.14 \%$ & $14.57 \%$ & $10.02 \%$ & $4.55 \% \%^{* *}$ \\
market rate & {$[265]$} & {$[149]$} & {$[80]$} & {$[55]$} & $p=0.0385$ \\
\hline
\end{tabular}

Number of observations in square brackets reflect the number of landlords that respond to an inquiry from neither, both, or only one of the groups. All tests of statistical significance are from McNemar paired difference in proportions tests, which follow a chi-square distribution. Apartment and Single Family status is taken from the Craigslist advertisement of each unit. Company listings are determined by the researchers' discretion. Advertised rents are compared to HUD FMRs based on city location and number of bedrooms.

** Significant at the $5 \%$ level.

Hispanics, or vice versa. To capture discrimination that occurs by the way that landlords respond to prospective tenants, we examine the content of each e-mail individually and categorize it as a positive, negative, or neutral response.

A positive response is any response that affirms the unit is available, provides an invitation for further contact (and provides further contact information like an address or phone number) or an invitation to view the unit, or attempts to clear up confusion about the unit in question (provides a list of available properties, for example). A negative response simply states that the unit is no longer available. A neutral response is one that responds with questions regarding what unit the potential tenant is interested in (without attempting to provide information), asks the tenant for more information about their housing requirements or current situation, or offers to call the potential tenant but does not provide their own contact information. We code each e-mail response as positive, negative, or neutral to examine if whites and Hispanics receive different responses, even from landlords that reply to both e-mails.

Table 11 shows the results of the difference between landlords responding positively or not to e-mail inquiries (positive responses are coded one, neutral and negative responses are coded zero). ${ }^{21}$ These results reinforce the finding shown in Table 5 that landlords treat e-mail

\footnotetext{
${ }^{21}$ We also examined if landlords were more likely to respond negatively to one group more often. These results do not show any statistically meaningful difference between whites and either type of Hispanic in our experiment. Only a very small number of e-mails ( 56 total) respond negatively to inquiries from either group.
} 
Table 11. Positive vs. Other or No Landlord Response and Tests for Discrimination

\begin{tabular}{lccccc}
\hline & Reply Neither & Reply Both & White Only & Hispanic Only & $\mathrm{H}_{\mathrm{O}}: \mathrm{R}_{\mathrm{W}}-\mathrm{R}_{\mathrm{H}}=0$ \\
\hline Audits with assimilated & & & & & \\
Hispanic names & & & & & \\
All audits & $55.73 \%$ & $19.72 \%$ & $12.93 \%$ & $11.62 \%$ & $1.31 \%$ \\
& {$[978]$} & {$[346]$} & {$[227]$} & {$[204]$} & $p=0.2893$ \\
High quality & $53.46 \%$ & $20.07 \%$ & $15.68 \%$ & $10.79 \%$ & $4.89 \% * *$ \\
& {$[317]$} & {$[119]$} & {$[93]$} & {$[64]$} & $p=0.0251$ \\
Low quality (1) & $57.45 \%$ & $18.00 \%$ & $11.82 \%$ & $12.73 \%$ & $-0.91 \%$ \\
& {$[316]$} & {$[99]$} & {$[65]$} & {$[70]$} & $p=0.7308$ \\
Low quality (2) & $56.37 \%$ & $20.92 \%$ & $11.27 \%$ & $11.44 \%$ & $-0.16 \%$ \\
& {$[345]$} & {$[128]$} & {$[69]$} & {$[70]$} & $p=1.0000$ \\
Audits with & & & & & \\
Nonassimilated & & & & & \\
Hispanic names & & $17.08 \%$ & $14.20 \%$ & $10.02 \%$ & $4.18 \% * * *$ \\
All audits & $58.69 \%$ & {$[225]$} & {$[187]$} & {$[132]$} & $p=0.0024$ \\
& {$[773]$} & $20.32 \%$ & $12.56 \%$ & $11.19 \%$ & $1.37 \%$ \\
High quality & $55.94 \%$ & {$[89]$} & {$[55]$} & {$[49]$} & $p=0.6241$ \\
& {$[245]$} & $16.08 \%$ & $14.45 \%$ & $10.26 \%$ & $4.20 \% *$ \\
Low quality (1) & $59.21 \%$ & {$[69]$} & {$[62]$} & {$[44]$} & $p=0.0982$ \\
& {$[254]$} & $14.89 \%$ & $15.56 \%$ & $8.67 \%$ & $6.89 \% * * *$ \\
Low quality (2) & $60.89 \%$ & {$[67]$} & {$[70]$} & {$[39]$} & $p=0.0039$ \\
& {$[274]$} & & & & \\
\hline
\end{tabular}

Number of observations in square brackets reflect the number of landlords that respond positively to an inquiry from neither, both, or only one of the groups. All tests of statistical significance are from McNemar paired difference in proportions tests, which follow a chi-square distribution. Assimilated Hispanics have a first name with $1<\mathrm{L}(\mathrm{W}) / \mathrm{L}(\mathrm{H})<$ $\infty$ and a single Hispanic surname. Nonassimilated Hispanics have a first name with $\mathrm{L}(\mathrm{H}) / \mathrm{L}(\mathrm{W})=\infty$ and two Hispanic surnames. High-quality e-mails are written formally with good grammar and no spelling mistakes. Low-quality type $1 \mathrm{e}-$ mails are less formal and contain a spelling mistake and grammatical errors. Low-quality type 2 e-mails are sent only by Hispanic auditors and contain grammatical errors common to native Spanish speakers that are not fluent in English; for these audits white tenants always send low-quality type 1 e-mails.

* Significant at the $10 \%$ level.

** Significant at the $5 \%$ level.

*** Significant at the $1 \%$ level.

inquiries from nonassimilated Hispanics differently than whites and in fact show larger levels of discrimination and are more statistically precise. Pooling all white/nonassimilated audits, we find that on net $4.18 \%$ of landlords (compared to $2.89 \%$ for the response/nonresponse), statistically significant at the $1 \%$ level, discriminate by responding positively to whites and either negatively or in a neutral way to Hispanics. We find the largest level of discrimination between whites and nonassimilated Hispanics when using low-quality e-mails, with net discrimination of $6.89 \%$ of landlords, statistically significant at the $1 \%$ level. In addition, we also find some evidence of discrimination when Hispanics do not use broken English, but have nonassimilated names, and for assimilated Hispanics, where both e-mails are higher quality. These results show that even among landlords that reply to both e-mails, they tend to send emails with a positive message to whites more often than to Hispanics.

The results in Table 11 depend on the subjective reading of e-mail content and accurate categorization into positive, negative, and neutral responses. While an objective criterion is the basis for this categorization, ultimately it is based on human judgment, which may be bias. Using broad categories also leaves out more subtle forms of discrimination, as explored by 
Hanson, Hawley, and Taylor (2011) against African Americans and highlighted by Ditlmann et al. (2010) as a potential margin of unequal treatment if overt discrimination is easily detectible. Although individuals who still feel racial or ethnic prejudice toward certain groups display these feelings less overtly, they may still manifest them in very real ways that are perceivable by the group being discriminated against. Often this form of discrimination is difficult to quantify and would not be captured in an online e-mail audit experiment if response rates were the only form of discrimination measured.

We attempt a more objective measure of response content and to capture more subtle forms of discrimination in several ways: first, through measuring the length of response the landlord sends; second, through the timing of the response, that is, if they send a reply slowly it may be a way to express lack of interest; and, last, through attempting to measure the quality of response using keyword searches of the text of landlord replies. We search for words that are associated with mentioning that the landlord has other units available (another, second, several), inviting the potential tenant to view the unit (view, tour, show, look, stop by, come by), and general polite language (thanks, please, call, sincerely, regards). ${ }^{22}$

Table 12 shows the results of testing for subtle forms of discrimination. This table shows how the content and timing of landlord responses differ between white and Hispanic tenants. We test if landlords use the type of language (invitation, mentioning other units) when replying to prospective tenants in the experiment. We find no evidence that landlords respond with longer replies to whites than to Hispanics, contrary to the findings in Hanson, Hawley, and Taylor (2011) for African Americans. We find strong evidence that landlords respond more slowly to whites than either Hispanic group — by as much as 64 minutes (1.08 hours). Similar to Hanson, Hawley, and Taylor (2011) we do find some evidence of differences in e-mail content between whites and minorities. The strongest finding is that landlords are much more likely to use polite language when responding to whites than they are when responding to nonassimilated Hispanics. These results suggest that landlords may practice discrimination by being more friendly or respectful with white clients, even if they do not overtly discriminate by not replying to Hispanic clients.

\section{Sample Representativeness and External Validity Concerns}

The design of our experiment ensures that the results we find are internally valid and represent the discrimination Hispanics would face if they searched for rental housing from a similar sample of units advertised on the internet. The design of the experiment does not ensure that our results are externally valid and representative of the discrimination Hispanics face in the larger rental housing market. We can think of at least two reasons why our results may not represent the discrimination Hispanics face in the larger rental housing market: (i) Hispanics may search for rental housing using different modes and (ii) our sample of rental units may not represent the rental units that Hispanics typically search for (either geographically, demographically, or by unit characteristics).

\footnotetext{
${ }^{22}$ We also searched for descriptive words (new, clean, quiet, nice, good), contact information (e-mail or phone number), greetings (hi, hello, dear), mentioning employment history (employment, job, employer, income, pay stub, paycheck), rental history (rental history, eviction), and background information (criminal, background, verify, credit check) and found no statistically significant differences in use of these words between whites and either group of Hispanics.
} 
Table 12. Content and Timing of Landlord Response and Tests for Discrimination

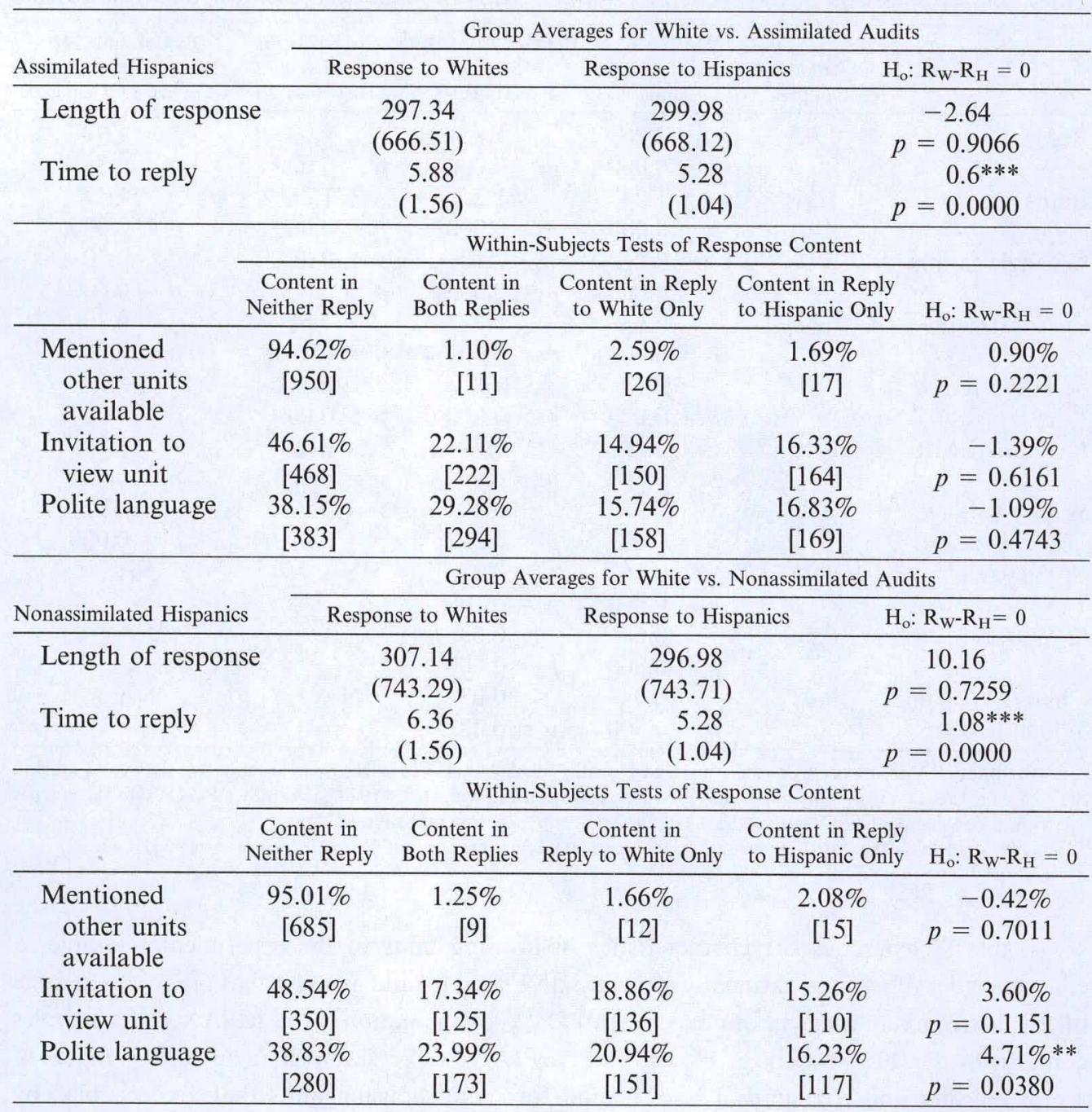

Standard deviations reported in parentheses. Number of observations in square brackets reflect the number of landlords that respond to an inquiry from neither, both, or only one of the groups using language from keyword searches. Tests for keyword searches of statistical significance are from McNemar paired difference in proportions tests, which follow a chi-square distribution. Length of Response and Time to Reply are averages for all e-mails from a given ethnicity in our experiment, and are not based on landlord response to one, neither, or both of our e-mails. Tests for difference in the time to reply and length of e-mail response are standard difference in means.

** Significant at the $5 \%$ level.

*** Significant at the $1 \%$ level.

Matching the characteristics of the larger market where Hispanics typically search may not be advantageous as minority renters may change search patterns if they believe they will be discriminated against, so in some sense finding discrimination in a more general sample is of interest. We do not have data on actual or hypothetical searches by Hispanic renters, so we cannot address these concerns directly. We can, however, compare our sample to a nationally representative sample of housing units that Hispanics actually rent. Comparing our results to units that are the outcome (where actual renters live) instead of preferred searches includes average differences in unit and neighborhood characteristics that result because discrimination occurs in the search for rental housing; nonetheless, it may shed light on any bias that could result from the experimental sample. 
Table 13. Comparison of Experimental Sample and Nationally Representative Housing Units

\begin{tabular}{|c|c|c|c|c|c|}
\hline & $\begin{array}{l}\text { Experimental } \\
\text { Sample }\end{array}$ & $\begin{array}{l}\text { All Rental } \\
\text { Units }\end{array}$ & $\begin{array}{l}\text { Rental Units for } \\
\text { MSAs in } \\
\text { Experiment }\end{array}$ & $\begin{array}{l}\text { All Rental Units } \\
\text { Occupied by } \\
\text { Hispanics }\end{array}$ & $\begin{array}{c}\text { Rental units for } \\
\text { MSAs in Experiment } \\
\text { Occupied by Hispanics }\end{array}$ \\
\hline \multirow[t]{2}{*}{ Beds } & 1.92 & 2.06 & 1.85 & 2.08 & 1.92 \\
\hline & & $p=0.000$ & $p=0.000$ & $p=0.000$ & $p=0.865$ \\
\hline \multirow[t]{2}{*}{ Baths } & 1.51 & 1.28 & 1.25 & 1.26 & 1.21 \\
\hline & & $p=0.000$ & $p=0.000$ & $p=0.000$ & $p=0.000$ \\
\hline \multirow[t]{2}{*}{$\%$ Single family } & 0.16 & 0.21 & 0.18 & 0.24 & 0.20 \\
\hline & & $p=0.000$ & $p=0.005$ & $p=0.000$ & $p=0.000$ \\
\hline \multirow[t]{2}{*}{ \% Apartment } & 0.68 & 0.65 & 0.71 & 0.65 & 0.70 \\
\hline & & $p=0.000$ & $p=0.002$ & $p=0.000$ & $p=0.047$ \\
\hline \multirow[t]{2}{*}{ Monthly rent } & $\$ 1516$ & $\$ 885$ & $\$ 1079$ & $\$ 867$ & $\$ 948$ \\
\hline & & $p=0.000$ & $p=0.000$ & $p=0.000$ & $p=0.000$ \\
\hline \multirow[t]{2}{*}{ \% Below FMR } & 0.41 & 0.79 & 0.77 & 0.89 & 0.89 \\
\hline & & $p \doteq 0.000$ & $p=0.000$ & $p=0.000$ & $p=0.000$ \\
\hline \multirow[t]{2}{*}{ Square footage } & 1163 & 1175 & 1135 & 1075 & 1042 \\
\hline & & $p=0.612$ & $p=0.223$ & $p=0.000$ & $p=0.000$ \\
\hline$\%$ Hispanic $^{\mathrm{a}}$ & 0.22 & 0.16 & 0.21 & NA & NA \\
\hline$\%$ White $^{\mathrm{a}}$ & 0.50 & $\begin{aligned} p= & 0.000 \\
& 0.64 \\
p= & 0.000\end{aligned}$ & $\begin{aligned} p= & 0.147 \\
& 0.68 \\
p= & 0.000\end{aligned}$ & NA & NA \\
\hline $\begin{array}{l}\text { Change Hispanic } \\
2000 \text { to } 2010^{\mathrm{a}}\end{array}$ & 0.03 & $p=0.000$ & $\begin{aligned} & 0.03 \\
p= & 0.422\end{aligned}$ & NA & NA \\
\hline
\end{tabular}

Rental unit samples are from the American Housing Survey (AHS) 2011 National Sample. We use rental units in the sample to create weighted averages that are representitive using weights by 1990 geography (WGT90GEO). $P$ values are from a one-sample $t$-test between the AHS value and the experimental sample.

${ }^{a}$ Indicates data from the 2010 Census not AHS; these characteristics are not sorted for rental housing units.

Table 13 compares the characteristics of housing units in the experimental sample to characteristics of various nationally representative samples and samples that are representative of the metropolitan areas in our experiment. Data for the national and MSA specific samples come from the 2011 American Housing Survey (AHS). We show characteristics samples of rental housing units (columns 1 and 2), and for rental housing units that are occupied by Hispanic tenants (columns 3 and 4).

Table 13 shows that there are many statistically significant differences between our sample and the AHS sample, but the magnitude of many of these differences is not large. For example, our sample has a slightly higher number of bathrooms on average and slightly fewer singlefamily homes. The largest difference between our sample and the AHS samples is for the rent and the units that rent below FMR. One reason the experiment sample rents are higher could be that our average is for an asked rent rather than a contract rent - once units are rented they may be contracted for less than asking price. Another reason is that the AHS is a sample of currently occupied units, some of which have long-term tenants whose rents may not have adjusted, or who signed longer-term leases. The percentage of units renting below FMR is lower in our sample both because our average rents are higher and because FMR in the AHS is reported based on the MSA average and is not based on the unit size as it is truly calculated; our measure considers the unit size (by number of bedrooms).

Most importantly, we can test to see if our experimental sample is driving the results we find for discrimination. If we reweight the sample based on rents that Hispanics actually pay 
(according to the national AHS data), we find nearly the same magnitude of discrimination as in the unweighted experiment. Weighting by rents yields a level of discrimination for the assimilated group of $-1.3 \%$ of landlords (not statistically significant), compared to $-1.8 \%$ for the unweighted sample. ${ }^{23}$ For the nonassimilated group, weighting yields a level of discrimination of $2.85 \%$ of landlords, compared to $2.89 \%$ for the unweighted sample.

If we reweight the sample based on the percentage of units that are below FMR according to the distribution in the AHS, we find a smaller level of discrimination against the nonassimilated Hispanics, but it is still statistically meaningful. Weighting by FMR yields a level of discrimination for the assimilated group of $-0.67 \%$, compared to $-1.8 \%$ for the unweighed sample. For the nonassimilated group, weighting by FMR yields a level of discrimination of $2 \%$ of landlords, compared to $2.89 \%$ for the unweighted sample. Reweighting by any of the other demographic and unit characteristic differences for which we have data yields even smaller differences in magnitude, so essentially no change in the primary results. It does not seem that the sample of units in our experiment is driving the results we find.

\section{Conclusion}

Our results uncover several key findings that add to the literature on housing discrimination. We find that Hispanics face different treatment in the housing market depending on how assimilated they appear to be to American culture. Overall, Hispanics with more Americanized names do not receive fewer responses than whites when inquiring to rent a housing unit. Treatment toward nonassimilated Hispanics is not equal, with 5.8\% of landlords discriminating when inquiries include broken English making the home seekers appear even more like recent immigrants. We also find discrimination in favor of white home seekers in the content of the response that shows landlords are more likely to send a positive reply and are more likely to use polite language.

The magnitude of discrimination toward nonassimilated Hispanics is slightly larger than the amount of discrimination uncovered by Hanson and Hawley (2011), who find that about $4.5 \%$ of landlords discriminate against African Americans. Finding discrimination against Hispanics is also consistent with the older literature using in-person audits, although the magnitudes are not comparable as not responding is not an option in these studies. Both the results presented here and in Hanson and Hawley (2011) show that U.S. housing markets have substantially fewer discriminating landlords than housing markets in Sweden (Ahmed and Hammarstedt 2008; Ahmed, Andersson, and Hammarstedt 2010) and in Spain (Bosch, Carnero, and Farre 2010).

Less favorable treatment toward Hispanics that appear to be recent immigrants may reduce their choices in finding housing that matches their needs. Many important local public services such as education are dependent upon the neighborhood in which one lives; therefore our research suggests that newly immigrated Hispanics may have a difficult time matching their preferences for local public services. This is particularly a problem for immigrants with children who may not have access to a quality education. Our results also show that Hispanics assimilated to American culture are more likely to be treated as equals to white Americans; if

\footnotetext{
${ }^{23}$ We assign weights based on quartile of the rent distribution for rents that Hispanics actually pay according to the AHS. The quartile breaks are $\$ 550, \$ 765$, and $\$ 1079$, respectively.
} 
nonassimilated Hispanics are kept out of white neighborhoods, this may slow cultural assimilation and prolong discrimination against this group.

The differences we find across regions, especially in the Southwest, indicate that enforcing Fair Housing Laws differentially may be a better use of resources. Although we do not have enough observations to definitively say what cities have the highest level of discrimination against Hispanics, shifting resources toward the Southwest region at the expense of other regions may net larger national gains (absent increased discrimination in those other regions if enforcement is reduced). Furthermore, finding discrimination with e-mail contact suggests that it is necessary to expand enforcement of Fair Housing Laws to online venues. Perhaps the most prudent policy recommendation is for more research on if, how, and why Hispanics are discriminated against in housing markets.

Future research on discrimination against Hispanics in the rental housing market might focus on the differences between different types of Hispanic groups. Our results give support to the idea that studies on Hispanics should use a multiple group structure, but it would also be interesting to study if discrimination happens when there is a match between the primary Hispanic culture (Mexican, Cuban, etc.) in the area and the person searching for housing. Additionally, further research might focus more intensely on the Southwest region where housing market discrimination against Hispanics is strongest as this may help to shed light on the cause of discrimination.

\title{
Appendix 1: E-Mail Text
}

\author{
High Quality
}

Greetings,

I was looking at your posting on Craigslist and I am interested in renting it. Is it still available? I have credit reports and references available upon request. Could you please also tell me a little more about the neighborhood? How is the safety and noise level? What kind of grocery stores are in the area? Thanks for your time.

Sincerely,

Hello,

I am moving soon and would like to know more about this area and the place you advertised. First of all, is it still for rent? Also what kind of amenities are in the area (parks, restaurants, etc.)? Let me know if you require references, credit reports, etc. and I can send that over. Thanks a lot.

Sincerely,

Low Quality (Type 1)

$\mathrm{Hi}$,

im looking for a new place and i saw your ad online. are you still renting it? What information do you need from me? i can get you it.

thanks,

Hey,

is the place you put on the internet availbe (sic) still? I am moving soon. If you want references let me know.

Thank You,

Low Quality (Type 2)

Hello,

I wanting to see your place. I need to make application? Please write me what I need you.

Thank You, 


\section{Appendix 2: Validating Assimilation Survey}

We conducted a survey to explore the assumption that the names and e-mail text in our survey convey information about the cultural assimilation of potential tenants. We surveyed 476 undergraduate students at a medium-sized, Midwestern, private university and asked them to indicate an opinion for each name and e-mail type in our original experiment. The actual survey instrument was a hard-copy two page handout with a cover page of instructions, as follows:

Instructions:

We are interested in your opinion about how a person's name and writing style may or may not reveal important information about them.

Your participation in this survey is voluntary, and will not affect your grade in this course in any way.

Do not write your name or any other personal information on the survey.

On the following page, you will see a list of names.

For each name, indicate if it conveys information about a person's familiarity with American customs and culture.

Please note you may choose the option "neutral" if you do not feel the name conveys any information about the person's familiarity with American customs and culture.

\section{Very Unfamiliar Neutral Familiar $\begin{gathered}\text { Very } \\ \text { Unfamiliar }\end{gathered}$
Familiar}

$\begin{array}{llllll}\text { Alex Lopez } & 0 & 0 & 0 & 0 & 0 \\ \text { Maxwell Davis } & 0 & 0 & 0 & 0 & 0 \\ \text { Anthony Gonzalez } & 0 & 0 & 0 & 0 & 0 \\ \text { Ruben Ramirez-Chacon } & 0 & 0 & 0 & 0 & 0 \\ \text { Brendan Miller } & 0 & 0 & 0 & 0 & 0 \\ \text { Zachary Larson } & 0 & 0 & 0 & 0 & 0 \\ \text { Andres Lozada-Alvarado } & 0 & 0 & 0 & 0 & 0 \\ \text { Jared Moore } & 0 & 0 & 0 & 0 & 0 \\ \text { Carlos Quintana } & 0 & 0 & 0 & 0 & 0 \\ \text { Tyler Anderson } & 0 & 0 & 0 & 0 & 0 \\ \text { Jonathan Santiago } & 0 & 0 & 0 & 0 & 0 \\ \text { Felix Villegas Ayala } & 0 & 0 & 0 & 0 & 0 \\ \text { Dylan Johnson } & 0 & 0 & 0 & 0 & 0 \\ \text { Manuel Medina Rios } & 0 & 0 & 0 & 0 & 0 \\ \text { Ethan Olson } & 0 & 0 & 0 & 0 & 0 \\ \text { Jack Wilson } & 0 & 0 & 0 & 0 & 0 \\ \text { Christian Garcia } & 0 & 0 & 0 & 0 & 0 \\ \text { Nathan Taylor } & 0 & 0 & 0 & 0 & 0 \\ \text { Oscar Nazario Ortiz } & 0 & 0 & 0 & 0 & 0 \\ \text { Brett Thomás } & 0 & 0 & 0 & 0 & 0\end{array}$


On the second page, you will see the text of different e-mail messages used to inquire about vacant rental apartments.

For each e-mail, indicate if it conveys information about the author's familiarity with American customs and culture.

Please note you may choose the option "neutral" if you do not feel the e-mail conveys any information about the person's familiarity with American customs and culture.

\section{Very Unfamiliar Neutral Familiar Very \\ Unfamiliar Unfamiliar Neutral Familiar Familiar}

$\mathrm{Hi}$,

im looking for a new place and $\mathrm{i}$ saw your ad online. are you still renting it? What infromation do you need from me? i can get it. thanks,

\section{Greetings,}

I was looking at your posting on

Craigslist and I am interested in renting it. Is it still available? I have credit reports and references available upon request. Could you please also tell me a little more about the neighborhood? How is the safety and noise level? What kind of grocery stores are in the area? Thanks for your time.

Sincerely,

Hello,

I wanting to see your place. I need to make application? Please write me what I need you.

Thank You,

The instructions were also read out loud for the participants, and they were assured that the survey was optional and did not have any bearing on their grades. To ensure confidentiality, students were instructed not to include their name or any other information on the response sheet.

Appendix Tables 1-4 summarize the results of the survey. Appendix Tables 1 and 3 show the overall proportion of respondents indicating how each name and e-mail was perceived. Appendix Tables 2 and 4 show regression results for linear probability and Probit regressions of the following form:

$$
\begin{gathered}
Y_{i, n}=\alpha+\sum_{n=1}^{19} \beta_{n}\left(D=1 \text { if } \text { Name }_{n}\right)+\delta_{i}+\varepsilon, \\
Y_{i, n}=\alpha+\sum_{t=1}^{3} \beta_{n}\left(D=1 \text { if } \text { Text }_{t}\right)+\delta_{i}+\varepsilon,
\end{gathered}
$$

where $i$ indicates the respondent, $n$ indicates the name in question, and $t$ indicates the text type. We estimate A1 and A2 with and without the fixed effect for individual respondents. An observation in these regressions consists of a response to a given name or text from an individual. 
Appendix Table 1. Survey Results for Relationship between Names and Cultural Assimilation Blank Very Unfamiliar Unfamiliar Neutral Familiar Very Familiar

White names

\begin{tabular}{lllllll} 
Maxwell Davis & $0.84 \%$ & $4.62 \%$ & $4.20 \%$ & $19.96 \%$ & $35.92 \%$ & $34.45 \%$ \\
Brendan Miller & $0.63 \%$ & $4.62 \%$ & $3.99 \%$ & $15.13 \%$ & $30.88 \%$ & $44.75 \%$ \\
Ethan Olson & $0.63 \%$ & $5.04 \%$ & $4.41 \%$ & $22.27 \%$ & $35.29 \%$ & $32.35 \%$ \\
Jack Wilson & $0.63 \%$ & $5.46 \%$ & $2.31 \%$ & $17.23 \%$ & $26.68 \%$ & $47.69 \%$ \\
Tyler Anderson & $0.42 \%$ & $5.88 \%$ & $2.31 \%$ & $17.44 \%$ & $25.63 \%$ & $48.32 \%$ \\
Nathan Taylor & $0.63 \%$ & $5.25 \%$ & $3.78 \%$ & $18.70 \%$ & $32.35 \%$ & $39.29 \%$ \\
Brett Thomas & $0.42 \%$ & $5.04 \%$ & $3.57 \%$ & $16.39 \%$ & $30.67 \%$ & $43.91 \%$ \\
Zachary Larson & $0.63 \%$ & $5.25 \%$ & $5.46 \%$ & $17.02 \%$ & $32.56 \%$ & $39.08 \%$ \\
Dylan Johnson & $0.63 \%$ & $5.46 \%$ & $4.20 \%$ & $15.97 \%$ & $31.30 \%$ & $42.44 \%$ \\
Jared Moore & $0.42 \%$ & $5.67 \%$ & $5.67 \%$ & $21.64 \%$ & $35.29 \%$ & $31.30 \%$ \\
Assimilated Hispanic names & & & & & & \\
Alex Lopez & $0.42 \%$ & $6.30 \%$ & $15.55 \%$ & $32.35 \%$ & $41.18 \%$ & $4.20 \%$ \\
Anthony Gonzalez & $1.68 \%$ & $7.56 \%$ & $18.49 \%$ & $34.03 \%$ & $33.82 \%$ & $4.41 \%$ \\
Jonathan Santiago & $1.05 \%$ & $7.77 \%$ & $17.65 \%$ & $35.50 \%$ & $32.98 \%$ & $5.04 \%$ \\
Christian Garcia & $0.42 \%$ & $7.56 \%$ & $16.18 \%$ & $32.56 \%$ & $36.34 \%$ & $6.93 \%$ \\
Carlos Quintana & $0.42 \%$ & $15.97 \%$ & $30.67 \%$ & $35.29 \%$ & $14.29 \%$ & $3.36 \%$ \\
Nonassimilated Hispanic & & & & & & \\
names & & & & & & \\
Ruben Ramirez Chacon & $1.05 \%$ & $22.90 \%$ & $34.87 \%$ & $28.78 \%$ & $9.45 \%$ & $2.94 \%$ \\
Felix Villegas Ayala & $0.42 \%$ & $26.47 \%$ & $32.98 \%$ & $27.10 \%$ & $10.71 \%$ & $2.31 \%$ \\
Oscar Nazario Ortiz & $0.63 \%$ & $19.96 \%$ & $31.09 \%$ & $28.99 \%$ & $15.76 \%$ & $3.57 \%$ \\
Manuel Medina Rios & $0.42 \%$ & $21.85 \%$ & $33.61 \%$ & $28.57 \%$ & $12.18 \%$ & $3.36 \%$ \\
Andres Lozada Alvarado & $0.42 \%$ & $23.95 \%$ & $35.50 \%$ & $26.89 \%$ & $10.08 \%$ & $3.15 \%$ \\
\hline
\end{tabular}

Survey is of 476 undergraduate students from a medium-sized, private, Midwestern university. Respondents were asked to indicate what they thought each name indicated about a person's familiarity with American customs and culture. 


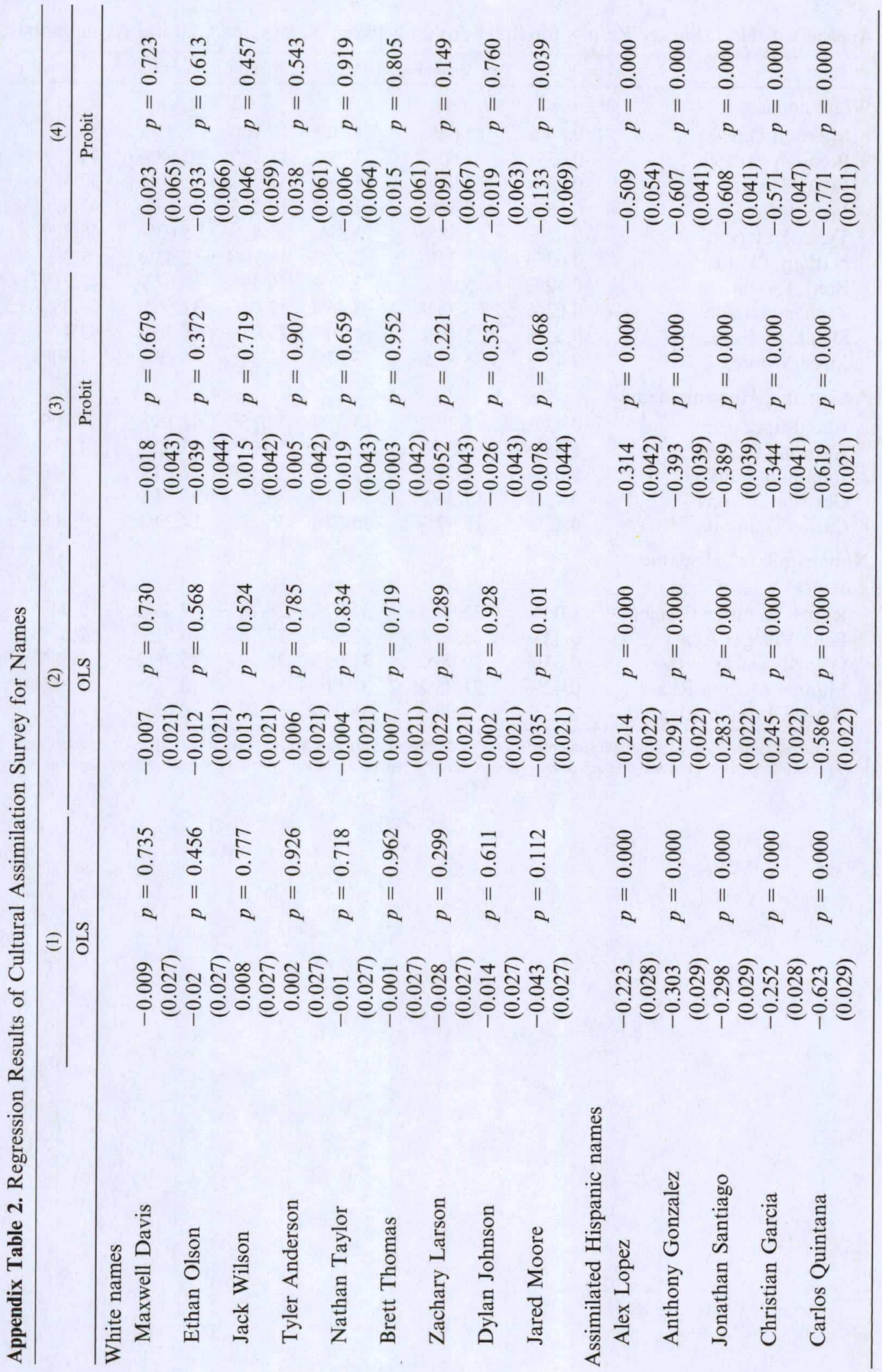




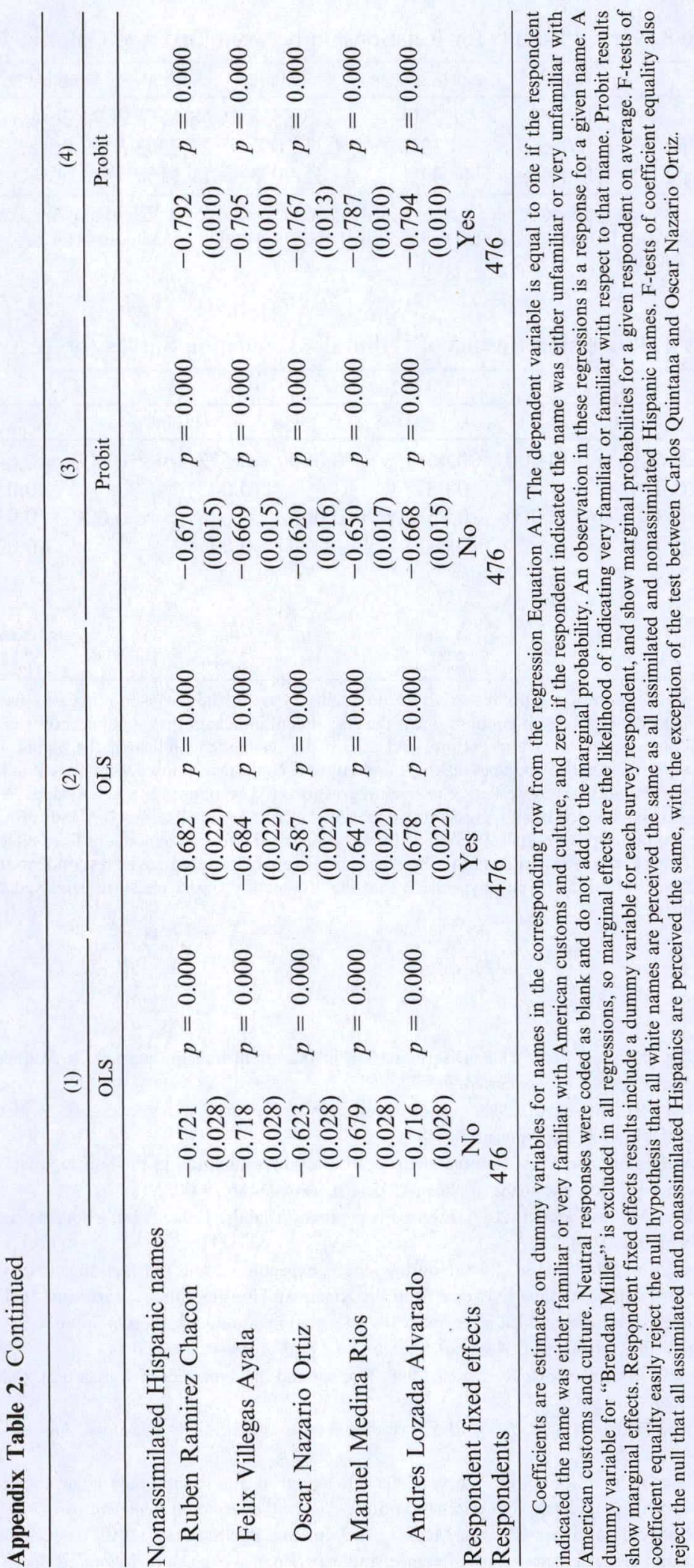


Appendix Table 3. Survey Results for Relationship between Text and Cultural Assimilation

\begin{tabular}{lcccrrc}
\hline & Blank & Very Unfamiliar & Unfamiliar & Neutral & Familiar & Very Unfamiliar \\
\hline High quality & $0.00 \%$ & $1.28 \%$ & $3.85 \%$ & $8.55 \%$ & $24.36 \%$ & $61.97 \%$ \\
Low quality type 1 & $0.00 \%$ & $8.12 \%$ & $31.20 \%$ & $23.93 \%$ & $29.06 \%$ & $7.69 \%$ \\
Low quality type 2 & $0.00 \%$ & $44.44 \%$ & $35.90 \%$ & $11.11 \%$ & $5.56 \%$ & $2.99 \%$ \\
\hline
\end{tabular}

Survey is of 234 undergraduate students from a medium-sized, private, Midwestern university. Respondents were asked to indicate what they thought each e-mail text indicated about the author's familiarity with American customs and culture.

Appendix Table 4. Regression Results of Cultural Assimilation Survey for Text

\begin{tabular}{|c|c|c|c|c|}
\hline & (1) & (2) & (3) & (4) \\
\hline & OLS & OLS & Probit & Probit \\
\hline $\begin{array}{l}\text { Low quality } \\
\text { type } 1\end{array}$ & $\begin{array}{c}-0.461 p=0.000 \\
(0.036)\end{array}$ & $\begin{array}{l}-0.465 p=0.000 \\
(0.037)\end{array}$ & $\begin{array}{l}-0.573 p=0.000 \\
(0.047)\end{array}$ & $\begin{array}{l}-0.642 p=0.000 \\
(0.051)\end{array}$ \\
\hline $\begin{array}{l}\text { Low quality } \\
\text { type } 2\end{array}$ & $\begin{array}{c}-0.848 p=0.000 \\
(0.034)\end{array}$ & $\begin{array}{c}-0.85 \\
(0.035)\end{array}$ & $\begin{array}{l}-0.828 p=0.000 \\
(0.027)\end{array}$ & $\begin{array}{l}-0.932 p=0.000 \\
(0.020)\end{array}$ \\
\hline $\begin{array}{l}\text { Respondent } \\
\text { fixed } \\
\text { effects }\end{array}$ & No & Yes & No & Yes \\
\hline Respondents & 234 & 234 & 234 & 234 \\
\hline
\end{tabular}

Coefficients are estimates on dummy variables for quality types in the corresponding row from the regression Equation A2. The dependent variable is equal to one if the respondent indicated the e-mail author was either familiar or very familiar with American customs and culture, and zero if the respondent indicated the e-mail author was either unfamiliar or very unfamiliar with American customs and culture. Neutral responses were coded as blank and do not add to the marginal probability. An observation in these regressions is a response for a given name. A dummy variable for the high-quality text is excluded in all regressions, so marginal effects are the likelihood of indicating very familiar or familiar with respect to that type of e-mail. Probit results show marginal effects. Respondent fixed effects results include a dummy variable for each survey respondent and show marginal probabilities for a given respondent on average. F-tests of coefficient equality easily reject the null hypothesis that the low-quality type 1 texts are perceived the same as highquality or low-quality type 2 .

\section{References}

Ahmed, Ali, and Mats Hammarstedt. 2008. Discrimination in the rental housing market: A field experiment on the internet. Journal of Urban Economics 64:362-72.

Ahmed, Ali, and Mats Hammarstedt. 2009. Detecting discrimination against homosexuals: Evidence from a field experiment on the internet. Economica 76:588-97.

Ahmed, Ali, Lina Andersson, and Mats Hammarstedt. 2010. Can discrimination in the housing market be reduced by increasing the information about the applicants? Land Economics 86:79-90.

Baldini, Massimo, and Marta Federici. 2011. Ethnic discrimination in the Italian rental housing market. Journal of Housing Economics 20:1-14.

Boehm, Thomas, and Alan Schlottmann. 2008. Housing tenure, expenditure, and satisfaction across Hispanic, AfricanAmerican, and white households: Evidence from the American Housing Survey. Cityscape 10:95-158.

Bosch, Mariano, Angeles Carnero, and Lídia Farré. 2010. Information and discrimination in the rental housing market: Evidence from a field experiment. Regional Science and Urban Economics 40:11-19.

Card, David, Alexandre Mas, and Jesse Rothstein. 2008. Tipping and the dynamics of segregation. Quarterly Journal of Economics 123:177-218.

Carpusor, Adrian, and William Loges. 2006. Rental discrimination and ethnicity in names. Journal of Applied Social Psychology 36:934-52.

Choi, Seok Joon, Jan Ondrich, and John Yinger. 2005. Do rental agents discriminate against minority customers? Evidence from the 2000 Housing Discrimination Study. Journal of Housing Economics 14:1-26.

Ditlmann, Ruth, John Dovidio, Agata Gluszek, Melissa-Sue John, and Paul Lagunes. 2010. Understanding bias toward Latinos: Discrimination, dimensions of difference, and experience of exclusion. Journal of Social Issues 66:59-78. 
Ewens, Michael, Bryan Tomlin, and Choon Wang. 2012. Statistical discrimination or prejudice? A large sample field experiment. Review of Economics and Statistics 96:135-50.

Fischer, Mary, and Douglas Massey. 2004. The ecology of racial discrimination. City and Community 3:221-41.

Hanson, Andrew, and Zackary Hawley. 2011. Do landlords discriminate in the rental housing market? Evidence from an internet field experiment in U.S. cities. Journal of Urban Economics 70:99-114.

Hanson, Andrew, Zackary Hawley, and Aryn Taylor. 2011. Subtle discrimination in the rental housing market: Evidence from e-mail correspondence with landlords. Journal of Housing Economics 20:276-84.

Heckman, James. 1998. Detecting discrimination. Journal of Economic Perspectives 12:101-16.

Heckman, James, and Peter Siegelman. 1993. The Urban Institute audit studies: Their methods and findings." In Clear and convincing evidence: Measurement of discrimination in America, edited by Michael E. Fix and Raymond $\mathrm{J}$. Struyk. Washington, DC: Urban Institute Press, pp. 187-258.

Lopez, Mark, and Daniel Dockterman. 2011. U.S. Hispanic country of origin counts for nation, top 30 metropolitan areas. Pew Hispanic Center Research Report, May 26.

Massey, Douglas, and Garvey Lundy. 2001. Use of Black English and Racial Discrimination in Urban Housing Markets: New Methods and Findings. Urban Affairs Review 48:452-69.

Midrigal, Lorena, Barbara Ware, Robyn Miller, German Saenz, Mario Chavez, and Darryl Dykes. 2001. Ethnicity, gene flow, and population subdivision in Limon, Costa Rica. American Journal of Physical Anthropology 114:99-108.

Ondrich, Jan, Stephen Ross, and John Yinger. 2003. Now you see it, now you don't: Why do real estate agents withhold available houses from Black customers. Review of Economics and Statistics 85:854-73.

Ondrich, Jan, Alex Stricker, and John Yinger. 1998. Do real estate brokers choose to discriminate? Evidence from the 1989 Housing Discrimination Study. Southern Economic Journal 64:880-901.

Page, Marianne. 1995. Racial and ethnic discrimination in urban housing markets: Evidence from a recent audit study. Journal of Urban Economics 38:183-206.

Ross, Stephen, and Margery Turner. 2005. Housing discrimination in metropolitan America: Explaining changes between 1989 and 2000. Social Problems 52:152-80.

Turner, Margery, Stephen Ross, George Galster, and John Yinger. 2002. Discrimination in metropolitan housing markets: Phase I-National results from Phase I of the HDS 2000. Washington, DC: U.S. Department of Housing and Urban Development.

Word, David, and Colby Perkins. 1996. Building a Spanish surname list for the 1990's - a new approach to an old problem. United States Census Bureau Working Paper No. 13.

Yinger John. 1986. Measuring racial discrimination with fair housing audits: Caught in the act. American Economic Review 76:881-93.

Zhao, Bo. 2005. Does the number of houses a broker shows depend on a home seeker's race? Journal of Urban Economics 57:128-47.

Zhao, Bo, Jan Ondrich, and Yinger. 2006. Why do real estate brokers continue to discriminate? Evidence from the 2000 Housing Discrimination Study. Journal of Urban Economics 59:394-419. 